TRANSACTIONS OF THE

AMERICAN MATHEMATICAL SOCIETY

Volume 357, Number 11, Pages 4301-4328

S 0002-9947(05)03956-5

Article electronically published on June 9, 2005

\title{
CLASSIFICATION PROBLEMS IN CONTINUUM THEORY
}

\author{
RICCARDO CAMERLO, UDAYAN B. DARJI, AND ALBERTO MARCONE
}

\begin{abstract}
We study several natural classes and relations occurring in continuum theory from the viewpoint of descriptive set theory and infinite combinatorics. We provide useful characterizations for the relation of likeness among dendrites and show that it is a bqo with countably many equivalence classes. For dendrites with finitely many branch points the homeomorphism and quasi-homeomorphism classes coincide, and the minimal quasihomeomorphism classes among dendrites with infinitely many branch points are identified. In contrast, we prove that the homeomorphism relation between dendrites is $S_{\infty}$-universal. It is shown that the classes of trees and graphs are both $\mathrm{D}_{2}\left(\boldsymbol{\Sigma}_{3}^{0}\right)$-complete, the class of dendrites is $\boldsymbol{\Pi}_{3}^{0}$-complete, and the class of all continua homeomorphic to a graph or dendrite with finitely many branch points is $\Pi_{3}^{0}$-complete. We also show that if $G$ is a nondegenerate finitely triangulable continuum, then the class of $G$-like continua is $\boldsymbol{\Pi}_{2}^{0}$-complete.
\end{abstract}

\section{INTRODUCTION}

This paper is devoted to the study of classes of continua and relations between continua from the viewpoint of descriptive set theory. The latter studies and classifies "definable" subsets of Polish (i.e. separable and completely metrizable) spaces. Our basic references are [Nad92] for continuum theory and [Kec95] for descriptive set theory.

As in Nad92, we will be concerned exclusively with continua that are metrizable, i.e. with metric continua. Hence a continuum is a metric space which is compact and connected. In particular every continuum is a Polish space. A continuum is nondegenerate if it contains at least two (and hence uncountably many) points. A subcontinuum is a continuum which is a subset of the space we are considering.

We start by explaining how descriptive set theory deals with (classes of) continua. If $X$ is a metrizable space, let $\mathrm{K}(X)$ be the space of all compact subsets of $X$ equipped with the Vietoris topology. Then, $\mathrm{K}(X)$ is metrizable and the operation $X \mapsto \mathrm{K}(X)$ preserves separability, complete metrizability and compactness ([Kec95, §4.F] or [Nad92, chapter IV]). In particular, if $X$ is Polish, so is $\mathrm{K}(X)$. Let $\mathrm{C}(X)$ consist of all elements of $\mathrm{K}(X)$ that are connected and nonempty, i.e. of all subcontinua of $X . \mathrm{C}(X)$ is closed in $\mathrm{K}(X)$ and, therefore, it is a Polish (resp. compact) space if $X$ is Polish (resp. compact).

Denote by $I$ the closed interval $[0,1]$. Every compact metric space (and hence in particular every continuum) is homeomorphic to a closed subset of the Hilbert cube

Received by the editors July 23, 2002.

2000 Mathematics Subject Classification. Primary 03E15, 54F15, 54H05; Secondary 06A07.

We thank the referee for making valuable suggestions which made the presentation of the paper clearer. 
$I^{\mathbb{N}}$. Hence $\mathrm{C}\left(I^{\mathbb{N}}\right)$ is a compact Polish space containing a homeomorphic copy of every continuum. Therefore, if $\mathcal{P}$ is a class of continua closed under homeomorphisms, it makes sense to identify $\mathcal{P}$ with the set (still denoted by $\mathcal{P}$ ) of all subcontinua of $I^{\mathbb{N}}$ belonging to $\mathcal{P}$. Now $\mathcal{P}$ is a subset of the Polish space $\mathrm{C}\left(I^{\mathbb{N}}\right)$ and can be studied with the tools and techniques of descriptive set theory. In particular, one can try to establish the position of $\mathcal{P}$ in the Borel and projective hierarchies. When this succeeds, it sheds some light on the complexity of $\mathcal{P}$ and gives lower limits for the complexity of any equivalent definition of the same class of continua.

We recall here some basic concepts from classical descriptive set theory (again, we refer to Kec95 for a complete treatment).

Let $X$ be a metrizable space and denote by $\boldsymbol{\Sigma}_{1}^{0}(X)$ the family of open subsets of $X$. For $1 \leq \alpha<\omega_{1}$ the classes $\boldsymbol{\Sigma}_{\alpha}^{0}(X)$ and $\boldsymbol{\Pi}_{\alpha}^{0}(X)$ of subsets of $X$ are defined by transfinite recursion: $\boldsymbol{\Pi}_{\alpha}^{0}(X)$ is the class of all complements of sets in $\boldsymbol{\Sigma}_{\alpha}^{0}(X)$, while, for $\alpha \geq 2, \Sigma_{\alpha}^{0}(X)$ is the family of countable unions $\bigcup_{n \in \mathbb{N}} A_{n}$ where $A_{n} \in$ $\boldsymbol{\Pi}_{\beta_{n}}^{0}(X)$ for some $\beta_{n}<\alpha$. This hierarchy (called the Borel hierarchy) is increasing $\left(\boldsymbol{\Sigma}_{\alpha}^{0}(X) \cup \boldsymbol{\Pi}_{\alpha}^{0}(X) \subseteq \boldsymbol{\Sigma}_{\beta}^{0}(X) \cap \boldsymbol{\Pi}_{\beta}^{0}(X)\right.$ whenever $\left.\alpha<\beta\right)$ and the union

$$
\mathbf{B}(X)=\bigcup_{1 \leq \alpha<\omega_{1}} \boldsymbol{\Sigma}_{\alpha}^{0}(X)=\bigcup_{1 \leq \alpha<\omega_{1}} \boldsymbol{\Pi}_{\alpha}^{0}(X)
$$

is the $\sigma$-algebra of Borel subsets of $X$. Sets in $\Pi_{2}^{0}$ are also called $G_{\delta}$.

A measurable space is standard Borel if it is equipped with the $\sigma$-algebra of Borel sets of a Polish topology. Any Borel subset of a Polish space with the $\sigma$-algebra of its Borel subsets is standard Borel.

Let $\boldsymbol{\Sigma}_{1}^{1}(X)$ be the family of analytic subsets of $X(A \subseteq X$ is analytic if it is the continuous image of a Polish space). For $n \geq 1$ the classes $\boldsymbol{\Sigma}_{n}^{1}(X)$ and $\boldsymbol{\Pi}_{n}^{1}(X)$ of subsets of $X$ are defined by recursion: $\boldsymbol{\Pi}_{n}^{1}(X)$ is the class of all complements of sets in $\boldsymbol{\Sigma}_{n}^{1}(X)$, while $\boldsymbol{\Sigma}_{n+1}^{1}(X)$ is the family of continuous images of $\boldsymbol{\Pi}_{n}^{1}$ sets. This hierarchy (called the projective hierarchy) is also increasing.

Let $\boldsymbol{\Gamma}$ be a class of sets in Polish spaces and let $\check{\Gamma}$ be its dual class, that is, the family of complements of elements of $\boldsymbol{\Gamma}$. The class $\mathrm{D}_{2}(\boldsymbol{\Gamma})$ is the family of sets of the form $A \backslash B$ where $A, B \in \boldsymbol{\Gamma}$. This is the same as the class of all intersections $A \cap B$ with $A \in \boldsymbol{\Gamma}, B \in \check{\boldsymbol{\Gamma}}$. The dual class of $\mathrm{D}_{2}(\boldsymbol{\Gamma})$ is the class $\check{\mathrm{D}}_{2}(\boldsymbol{\Gamma})$ of all unions $A \cup B$ with $A \in \boldsymbol{\Gamma}, B \in \check{\boldsymbol{\Gamma}}$. As the subscript 2 indicates, this notion can be further extended (even in the transfinite), obtaining the difference hierarchy relative to $\boldsymbol{\Gamma}$.

If $X$ and $Y$ are metrizable spaces, $A \subseteq X, B \subseteq Y$, we say that $A$ is Wadge reducible to $B$ (we write $A \leq_{\mathrm{w}} B$ ) if $A=f^{-1}(B)$ for some continuous function $f: X \rightarrow Y$. Notice that if e.g. $B$ is $\boldsymbol{\Sigma}_{\alpha}^{0}$ and $A \leq_{\mathrm{W}} B$, then $A$ is also $\boldsymbol{\Sigma}_{\alpha}^{0}$. Thus, proving that $A \leq_{\mathrm{w}} B$ for some $A$ of known complexity yields a lower bound on the complexity of $B$.

If $\boldsymbol{\Gamma}$ is a class of sets in Polish spaces (like the classes $\boldsymbol{\Sigma}_{\alpha}^{i}$ and $\boldsymbol{\Pi}_{\alpha}^{i}$ introduced above), $Y$ is a Polish space and $A \subseteq Y$, say that $A$ is $\boldsymbol{\Gamma}$-hard if $B \leq_{\mathrm{W}} A$ for any $B \in \boldsymbol{\Gamma}(X)$ where $X$ is a zero-dimensional Polish space. Say that $A$ is $\boldsymbol{\Gamma}$-complete if, in addition, $A \in \boldsymbol{\Gamma}(Y)$.

It turns out that, for any $\alpha$, a set is $\boldsymbol{\Sigma}_{\alpha}^{0}$-complete if and only if it is $\boldsymbol{\Sigma}_{\alpha}^{0}$ but not $\boldsymbol{\Pi}_{\alpha}^{0}$, and similarly interchanging $\boldsymbol{\Sigma}_{\alpha}^{0}$ and $\boldsymbol{\Pi}_{\alpha}^{0}$. Analogously, a set is $\mathrm{D}_{2}\left(\boldsymbol{\Sigma}_{\alpha}^{0}\right)$ complete if and only if it is $\mathrm{D}_{2}\left(\boldsymbol{\Sigma}_{\alpha}^{0}\right)$ but not $\check{\mathrm{D}}_{2}\left(\boldsymbol{\Sigma}_{\alpha}^{0}\right)$. 
In this paper we investigate from the viewpoint of the Borel and projective hierarchies several natural classes of continua. Here are the definitions (we adhere to the terminology of $[\mathrm{Nad} 92])$.

Definition 1.1. An arc is a continuum homeomorphic to the closed interval $I$. If $A$ is an arc and $h: I \rightarrow A$ is a homeomorphism, the end points of $A$ are $h(0)$ and $h(1)$ (it is easy to see that the end points do not depend on the homeomorphism).

A graph is a continuum which can be written as the union of finitely many arcs which pairwise intersect only in their end points (none, one or both).

A tree is a graph which contains no subcontinuum homeomorphic to the circle $S^{1}$.

A Peano continuum is a continuum which is locally connected or, equivalently, which is the continuous image of $I$.

A dendrite is a Peano continuum which contains no subcontinuum homeomorphic to the circle $S^{1}$.

A continuum $X$ is arcwise connected if for all $x, y \in X$ with $x \neq y$ there exists an arc contained in $X$ with end points $x$ and $y$.

A continuum $X$ is uniquely arcwise connected if for all $x, y \in X$ with $x \neq y$ there exists a unique arc contained in $X$ with end points $x$ and $y$.

A continuum is indecomposable if it cannot be written as the union of two proper subcontinua. Otherwise it is decomposable.

A continuum is irreducible between $n$ points $(n \geq 2)$ if it contains a set of $n$ points which is not contained in any proper subcontinua. When $n=2$ we say that the continuum is irreducible.

A continuum is unicoherent if whenever it is written as the union of two subcontinua, then their intersection is a continuum.

A continuum is hereditarily indecomposable if all its nondegenerate subcontinua are indecomposable. Analogously we define hereditarily decomposable, hereditarily irreducible and hereditarily unicoherent continua.

A continuum is a dendroid if it is arcwise connected and hereditarily unicoherent.

A continuum is a $\lambda$-dendroid if it is hereditarily decomposable and hereditarily unicoherent.

Note that every graph (and hence every tree) is a Peano continuum. Therefore, trees are dendrites. Dendrites are uniquely arcwise connected. Since $I$ is the continuous image of every nondegenerate continuum, every Peano continuum has this property.

We also investigate properties such as being homeomorphic to a given continuum. In this case we are considering equivalence classes with respect to the homeomorphism equivalence relation on the space $\mathrm{C}\left(I^{\mathbb{N}}\right)$, and we exploit some results belonging to the rich subject of "Borel reducibility for equivalence relations" (see e.g. BK96], Hjo00] and [Kec02]). Here we only give the basic definitions we will need.

If $E$ and $F$ are equivalence relations on the standard Borel spaces $X$ and $Y$ respectively, a reduction of $E$ to $F$ is a function $f: X \rightarrow Y$ such that

$$
\forall x_{0}, x_{1} \in X\left(x_{0} E x_{1} \Longleftrightarrow f\left(x_{0}\right) F f\left(x_{1}\right)\right) .
$$

If $f$ is Borel this means that $E$ is Borel reducible to $F$.

For $\mathcal{L}$ a countable relational language, let $X_{\mathcal{L}}$ be the Polish space of (codes for) $\mathcal{L}$ structures with universe $\mathbb{N}$ (see Kec95 $\$ 16 . \mathrm{C}]$ ). If $E$ is an equivalence relation on a 
standard Borel space, $E$ is classifiable by countable structures if it is Borel reducible to isomorphism on $X_{\mathcal{L}}$ for some $\mathcal{L}$; it is $S_{\infty}$-universal if, in addition, isomorphism on $X_{\mathcal{L}}$ is Borel reducible to $E$ for every $\mathcal{L}$. If $E$ is a $S_{\infty}$-universal equivalence relation on a standard Borel space $X$, then for every Polish space in which $X$ is embedded as a Borel subset there exists a perfect set of pairwise non-equivalent elements.

Definition 1.2. If $C$ is a continuum, let

$$
\mathcal{H}(C)=\left\{X \in \mathrm{C}\left(I^{\mathbb{N}}\right) \mid X \text { is homeomorphic to } C\right\} .
$$

If $C$ is a nondegenerate continuum it is well known that $\mathcal{H}(C)$ is dense in $\mathrm{C}\left(I^{\mathbb{N}}\right)$.

Theorem 1.3. $\mathcal{H}(C)$ is Borel for every continuum $C$. For every $\alpha<\omega_{1}$, there exists a continuum $C$ such that $\mathcal{H}(C)$ is not $\mathbf{\Pi}_{\alpha}^{0}$, i.e. $\mathrm{C}\left(I^{\mathbb{N}}\right)$ is partitioned into homeomorphism classes of unbounded Borel complexity.

Proof. Each $\mathcal{H}(C)$ is Borel by a result proved in RN65.

The homeomorphism relation on $\mathrm{K}\left(2^{\mathbb{N}}\right)$ is $S_{\infty}$-universal by a result in [CG01. Theorem 4 of [FS89] (in that paper $S_{\infty}$-universality is called Borel completeness) implies that this equivalence relation is $\boldsymbol{\Sigma}_{1}^{1}$-complete (and hence not Borel) as a subset of $\mathrm{K}\left(2^{\mathbb{N}}\right) \times \mathrm{K}\left(2^{\mathbb{N}}\right)$. By a folklore result (see [BK96. Theorem 7.1.1]) homeomorphism classes in $\mathrm{K}\left(2^{\mathbb{N}}\right)$ have unbounded complexity in the Borel hierarchy.

We now Borel (in fact continuously) reduce homeomorphism on non-singleton compact subsets of $2^{\mathbb{N}}$ to homeomorphism on $\mathrm{C}\left(I^{2}\right)$ (and a fortiori on $\mathrm{C}\left(I^{\mathbb{N}}\right)$ ), which implies that homeomorphism classes of continua have unbounded complexity in the Borel hierarchy. To this end, view $2^{\mathbb{N}}$ as a subset of $I \times\{0\}$, let $p=(0,1)$ and to each $K \in \mathrm{K}\left(2^{\mathbb{N}}\right)$ associate the union of all straight segments joining any $x \in K$ with $p$ (this is called the cone on $K$ ). Two compact subsets of $2^{\mathbb{N}}$ of cardinality at least 2 are homeomorphic if and only if their cones are homeomorphic.

We point out that the argument in Hjo00 §4.3] shows that the homeomorphism relation between continua is strictly more complicated than $S_{\infty}$-universal ones (Hjorth actually mentions compacta, but his construction provides Peano continua). Corollary 8.6 and Theorem 8.7 compute the exact complexity of some $\mathcal{H}(C)$ 's, Theorem 6.7 shows that homeomorphism on dendrites is $S_{\infty}$-universal, while Corollary 6.8 sharpens the second part of Theorem 1.3 .

An important tool in continuum theory is the relation of likeness. It naturally leads to another equivalence relation, whose study is useful in obtaining our classification results.

Definition 1.4. If $\mathcal{C}$ is a class of continua and $X$ is a continuum, we say that $X$ is $\mathcal{C}$-like if for every $\varepsilon>0$ there exists $Y \in \mathcal{C}$ and a continuous map $f: X \rightarrow Y$ such that $f$ is onto and $\{x \in X \mid f(x)=y\}$ has diameter less than $\varepsilon$ for each $y \in Y$. The map $f$ is called an $\varepsilon$-map.

When $\mathcal{C}=\{Y\}$ we say that $X$ is $Y$-like and write $X \preceq Y$. $X \prec Y$ means $X \preceq Y$ and $Y \npreceq X$. Two continua $X$ and $Y$ are quasi-homeomorphic if $X \preceq Y$ and $Y \preceq X$.

It is immediate that $\preceq$ is transitive and reflexive (but not antisymmetric), i.e. a quasi-ordering, and hence the quasi-homeomorphism relation is an equivalence relation. Since likeness is invariant under homeomorphism, quasi-homeomorphism is coarser than homeomorphism. 
Definition 1.5. If $C$ is a continuum, let

$$
\begin{aligned}
\mathcal{Q}(C) & =\left\{X \in \mathrm{C}\left(I^{\mathbb{N}}\right) \mid X \text { is quasi-homeomorphic to } C\right\} \quad \text { and } \\
\mathcal{L}_{C} & =\left\{X \in \mathrm{C}\left(I^{\mathbb{N}}\right) \mid X \preceq C\right\} .
\end{aligned}
$$

Similarly, when $\mathcal{C}$ is a class of continua, we write $\mathcal{L}_{\mathcal{C}}$ for the set of all $\mathcal{C}$-like continua.

Since $\mathcal{L}_{C} \supseteq \mathcal{Q}(C) \supseteq \mathcal{H}(C)$, when $C$ is nondegenerate both $\mathcal{L}_{C}$ and $\mathcal{Q}(C)$ are dense in $\mathrm{C}\left(I^{\mathbb{N}}\right)$.

We will use and extend the following results of Kato and Ye ( $\mathrm{KY} 00]$ ).

Theorem 1.6. If $G$ is a graph, then $\mathcal{Q}(G)=\mathcal{H}(G)$.

Theorem 1.7. Let $P$ be a Peano continuum and let $G$ be a graph. Then $P \preceq G$ if and only if $P$ is a graph obtained from $G$ by shrinking to points finitely many pairwise disjoint subgraphs. In particular, if $G$ is a tree, then $P$ is a tree as well.

Theorem 1.7 can be rephrased in terms of monotone maps.

Definition 1.8. A continuous map between continua is monotone if the preimage of every point in the range is a continuum or, equivalently, the preimage of every subcontinuum of the range is a continuum.

Hence Theorem 1.7 says that if $P$ is a Peano continuum and $G$ is a graph, then $P \preceq G$ if and only if there exists a monotone map from $G$ onto $P$ such that only finitely many preimages of points are nondegenerate. In turn this is equivalent to the existence of a monotone map from $G$ onto $P$. The last equivalence follows from the fact $G$ has only finitely many branch points and each branch point is of finite order (see Definition 2.3).

We now explain the organization of the paper and announce our main results.

In Section 2 we introduce some notation and terminology about continua (mostly taken from [Nad92], and we refer to this book for proofs and more details). The reader can skip this section and refer back to it as needed.

In Section 3 we study the relation of likeness between dendrites. Since the notion of likeness is very important in continuum theory, this is interesting for its own sake, but the results of this section will also be instrumental in obtaining classification results for important classes of continua in the following sections. We characterize likeness between dendrites in Theorem 3.2, which improves Theorem 1.7 and shows that to establish whether a dendrite is like another it suffices to check likeness between their subtrees. We also extend Theorem 1.6 by showing in Theorem 3.5 that for dendrites with finitely many branch points, homeomorphism and quasi-homeomorphism coincide. This does not hold for arbitrary dendrites, and in Theorem 3.7 the minimal (with respect to likeness) quasi-homeomorphism classes among dendrites with infinitely many branch points are identified.

In Section 4, the characterization provided by Theorem 3.2 leads to combinatorial results: we investigate the structure of the partial order of likeness among dendrites, showing that it is bqo (in particular well-founded with no infinite antichains) and proving in Theorem 4.7 that there are countably many classes of quasi-homeomorphism among dendrites.

In Section 5 we show that the class of $\mathcal{C}$-like continua is $\boldsymbol{\Pi}_{2}^{0}$-complete for any nonempty collection $\mathcal{C}$ of nondegenerate dendrites or finitely triangulable continua (and indeed of even more general continua). This allows the use of Baire Category 
arguments to show e.g. that the pseudoarc is $G$-like for every nondegenerate finitely triangulable continuum $G$.

In Section 6] we solve the classification problem for homeomorphism on dendrites, by showing in Theorem 6.7 that it is $S_{\infty}$-universal. (In the same vein, Marcone and Rosendal in MR04 proved that the equivalence relation of continuous mutual embeddability among dendrites is much more complicated than $S_{\infty}$-universal.) $S_{\infty}$ universality actually already holds for one of the simplest quasi-homeomorphism classes of dendrites which is not a homeomorphism class. Therefore, as soon as dendrites are so complex that homeomorphism and quasi-homeomorphism do not coincide, a single class of quasi-homeomorphic dendrites may contain uncountably many classes of homeomorphism whose complexity lies arbitrarily high in the Borel hierarchy. Thus quasi-homeomorphism is much coarser than homeomorphism even among dendrites.

Section 7 introduces some constructions which are basic in obtaining lower bounds for the complexity of Borel subsets of $\mathrm{C}\left(I^{\mathbb{N}}\right)$ with the technique of Wadge reduction.

Section 8 contains the classification results for many classes of continua (a few more results of this kind, including $\boldsymbol{\Pi}_{1}^{1}$-completeness of hereditarily locally connected continua, are proved in DM04 ). Though we state our results in $\mathrm{C}\left(I^{\mathbb{N}}\right)$, they hold in $\mathrm{C}\left(I^{n}\right)$ for any $n \geq 2$. The classes of Peano continua and dendrites are $\boldsymbol{\Pi}_{3}^{0}$-complete, while those of graphs and trees are $\mathrm{D}_{2}\left(\boldsymbol{\Sigma}_{3}^{0}\right)$-complete. The latter results are interesting from a purely descriptive set-theoretic viewpoint as well, since there are few known natural sets whose sharp classification involves the difference hierarchy. We also show that the quasi-homeomorphism class of any nondegenerate dendrite or graph is $\boldsymbol{\Pi}_{3}^{0}$-complete.

\section{Notation AND TERMinOlogy}

First of all for any metrizable space $X$ (such as $I^{\mathbb{N}}$, or any continua) we fix a compatible bounded metric $d$. Any $A \subseteq X$ has a diameter with respect to $d$, which we write $\operatorname{diam}(A)$. Using $d$ we can define the Hausdorff metric $d_{H}$ on $\mathrm{K}(X)$, and hence on $\mathrm{C}(X)$ : $d_{H}$ is compatible with the Vietoris topology.

Definition 2.1. Let $X$ be a continuum and let $X_{1}, \ldots, X_{n}$ be subcontinua of $X$. $X$ is the essential sum of the $X_{i}$ 's (we write $X=\bigoplus_{i=1}^{n} X_{i}$ ) if $X=\bigcup_{i=1}^{n} X_{i}$ and $X \neq \bigcup_{i \neq j} X_{i}$ for every $j=1, \ldots, n$.

Definition 2.2. If $X$ is a continuum and $x \in X$, then the order of $x$ in $X$, denoted by $\operatorname{ord}(x, X)$, is the smallest cardinal number $\kappa$ such that there exists a neighborhood-base for $x$ in $X$ consisting of open sets each with boundary of cardinality less than or equal to $\kappa$.

If $G$ is a graph, then every point of $G$ has finite order in $G$. If $D$ is a dendrite, then every point of $D$ has order at most $\aleph_{0}$ in $D$.

Definition 2.3. If $X$ is a continuum, let $B(X)=\{x \in X \mid \operatorname{ord}(x, X)>2\}$ and $E(X)=\{x \in X \mid \operatorname{ord}(x, X)=1\} . E(X)$ is the set of end points of $X$ (when $X$ is an arc this definition coincides with the one given above) and $B(X)$ is the set of branch points of $X$.

If $G$ is a graph, then $B(G)$ and $E(G)$ are both finite. If $D$ is a dendrite, then $B(D)$ is countable, but $E(D)$ can be uncountable. 
Definition 2.4. A simple triod is a continuum homeomorphic to $(I \times\{0\}) \cup$ $\left(\left\{\frac{1}{2}\right\} \times I\right)$.

A comb is a tree $T$ such that $B(T) \subset A \subset T$ for some $\operatorname{arc} A$ and $\operatorname{ord}(x, T)=3$ for every $x \in B(T)$.

Note that arcs and simple triods are combs.

The following definition links our graphs (which are continua) with the objects studied in graph theory.

Definition 2.5. Let $G$ be a graph. A subdivision of $G$ is a pair $\Sigma=(N, E)$ where $N$ is a finite set of points (called nodes) of $G$ and $E=\left\{A_{1}, \ldots, A_{n}\right\}$ is a finite set of subarcs (called edges) of $G$ such that $G=\bigoplus_{i=1}^{n} A_{i}, N$ is the set of all end points of the elements of $E$ and the elements of $E$ pairwise intersect only in at most one of their end points.

It is immediate that every graph has (infinitely many) subdivisions. If we look at trees there is a canonical subdivision.

Definition 2.6. We denote by $\Sigma_{T}=\left(N_{T}, E_{T}\right)$ the unique subdivision of the tree $T$ such that $N_{T}=E(T) \cup B(T)$.

Definition 2.7. Let $G$ be a graph, $\Sigma=(N, E)$ a subdivision of $G$ and $\varepsilon>0$. We say that $\varepsilon$ separates $\Sigma$ if the following properties hold:

(1) each node of $N$ is more than $\varepsilon$ apart from each other node;

(2) each node of $N$ is more than $\varepsilon$ apart from each edge of $E$ which it does not belong to;

(3) any two disjoint edges of $E$ are more than $\varepsilon$ apart.

We note that for every graph $G$ and every $\Sigma$, there is $\varepsilon>0$ which separates $\Sigma$.

Definition 2.8. If $C$ is a continuum an open free arc of $C$ is any open subset of $C$ of the form $A \backslash\{x, y\}$, where $A$ is a subarc with end points $x$ and $y$.

Definition 2.9. If the continuum $X$ is uniquely arcwise connected and $x \neq y$ are in $X$ we denote the unique arc contained in $X$ with end points $x$ and $y$ by $A_{x y}^{X}$. It is also convenient to define $A_{x x}^{X}=\{x\}$.

The following theorem lists some basic facts about dendrites which we will use frequently and at times implicitly.

Theorem 2.10. Let $D$ be a dendrite. Then:

(1) every subcontinuum of $D$ is a dendrite;

(2) if $X$ is a subcontinuum of $D$, and $C$ is a connected component of $D \backslash X$, then $C$ is open in $D$ and $\bar{C} \backslash C$ has exactly one point;

(3) $D$ is hereditarily unicoherent, i.e. if the intersection of two subcontinua of $D$ is nonempty, then it is a subcontinuum of $D$.

Definition 2.11. If $D$ is a dendrite and $X$ is a subcontinuum of $D$, for any $x \in D$ there exists a unique $r(x) \in X$ which belongs to any $A_{x y}^{D}$ with $y \in X$. The function $r: D \rightarrow X$ is continuous and is called the first point map for $X$. In addition, using Theorem 2.10(2), it can be shown that this map is monotone.

Definition 2.12. If $D$ is a dendrite and $F$ is a finite subset of $D$ of cardinality at least 2, the smallest subtree of $D$ containing $F$ is $\bigcup_{x, y \in F} A_{x y}^{D}$, which is indeed a tree. 
Definition 2.13. If $D$ is a dendrite, an approximating sequence for $D$ is a sequence $\left(T_{n}\right)$ of subtrees of $D$ such that $\lim T_{n}=D$ (the limit being taken in the Vietoris topology).

Every nondegenerate dendrite has approximating sequences. Moreover, the following holds.

Theorem 2.14. Let $X$ be a dendrite and let $\left(T_{n}\right)$ be an approximating sequence of $X$. Let $r_{n}$ be the first point map for $T_{n}$. Then, $\left(r_{n}\right)$ converges uniformly to the identity map on $X$. Moreover, if $\varepsilon>0$, then for sufficiently large $n, r_{n}$ is an $\varepsilon$-map from $X$ onto $T_{n}$.

\section{LIKENESS BETWEEN DENDRITES}

We are now going to characterize likeness on dendrites.

Lemma 3.1. Let $X$ be a dendrite and let $T \subseteq X$ be a tree. Then $T \preceq X$.

Proof. Let $r: X \rightarrow T$ be the first point map for $T$. Fix $\varepsilon>0$ and let $T=\bigoplus_{j=1}^{n} A_{j}$, where the $A_{j}$ 's are arcs and two such arcs meet at most at an end point of both. We may assume that $\operatorname{diam}\left(A_{j}\right)<\frac{\varepsilon}{2}$ for each $j$. Let $X_{j}=r^{-1}\left(A_{j}\right)$. Then, $A_{j} \subseteq X_{j}$. As $r$ is monotone, $X_{j}$ is a continuum and hence a dendrite. Since $T=\bigoplus_{j=1}^{n} A_{j}$, $X=\bigoplus_{j=1}^{n} X_{j}$. Since $r$ is a first point map, we also have that $A_{i} \cap A_{j} \neq \emptyset$ if and only if $X_{i} \cap X_{j} \neq \emptyset$.

For every $j$, let $f_{j}$ be a continuous function from $A_{j}$ onto $X_{j}$ which is the identity on the end points of $A_{j}$. Then, $\bigcup_{j=1}^{n} f_{j}$ is an $\varepsilon$-map from $T$ onto $X$.

Theorem 3.2. Let $X$ be a nondegenerate Peano continuum and $Y$ be a nondegenerate dendrite. Then the following are equivalent:

(1) $X \preceq Y$;

(2) $X$ is a dendrite and $T \preceq Y$ for each tree $T \subseteq X$;

(3) $X$ is a dendrite and there exists an approximating sequence $\left(T_{n}\right)$ for $X$ such that $T_{n} \preceq Y$ for every $n$;

(4) $X$ is a dendrite and for every tree $T \subseteq X$ there exists a tree $V \subseteq Y$ such that $T \preceq V$;

(5) $X$ is a dendrite and for each tree $T \subseteq X$ there exists a monotone map from $Y$ onto $T$.

Proof. (1) $\Rightarrow$ (2) A Peano continuum is a dendrite if and only if it is tree-like [Nad92, exercise 10.50]. Therefore $Y$ is tree-like and, since $X \preceq Y$, so is $X$. Thus $X$ is a dendrite. If $T \subseteq X$ is a tree, then $T \preceq X$ by Lemma 3.1 and hence $T \preceq Y$.

$(2) \Rightarrow(3)$ Let $\left(T_{n}\right)$ be an approximating sequence for $X$. By (2), $T_{n} \preceq Y$ for every $n$.

(3) $\Rightarrow(1)$ Let $\varepsilon>0$. We want to find an $\varepsilon$-map from $X$ onto $Y$. By Theorem 2.14 there is $n$ such that $r_{n}: X \rightarrow T_{n}$, the first point map of $T_{n}$, is an $\varepsilon$-map. Let $\eta>0$ be such that if $M \subseteq T_{n}$ has diameter less than $\eta$, then $\operatorname{diam}\left(r_{n}^{-1}(M)\right)<\varepsilon$. Since $T_{n} \preceq Y$, there is an $\eta$-map $f$ from $T_{n}$ onto $Y$. Then, $f \circ r_{n}: X \rightarrow Y$ is the desired $\varepsilon$-map.

$(2) \Rightarrow(4)$ Let $T \subseteq X$ be a tree and let $\varepsilon>0$ separate $\Sigma_{T}$. Let $f$ be an $\varepsilon$ map from $T$ onto $Y$. Let $V \subseteq Y$ be the smallest tree of $Y$ containing $f\left(N_{T}\right)$. We claim that $T \preceq V$. Let $J=\left\{(p, q) \in N_{T} \times N_{T} \mid A_{p q}^{T} \in E_{T}\right\}$ and note that $V=\bigcup_{(p, q) \in J} A_{f(p) f(q)}^{Y}$. 
Fix $(p, q) \in J$. First, note that $A_{f(p) f(q)}^{Y} \subseteq f\left(A_{p q}^{X}\right)$. If $p \in E(T)$ let $B_{p q}=\{f(p)\}$, while if $p \notin E(T)$ let

$$
B_{p q}=\bigcup_{\substack{\left(p, q^{\prime}\right) \in J \\ q^{\prime} \neq q}} A_{f(p) f(q)}^{Y} \cap A_{f(p) f\left(q^{\prime}\right)}^{Y}
$$

By Theorem 2.10 (3) each $A_{f(p) f(q)}^{Y} \cap A_{f(p) f\left(q^{\prime}\right)}^{Y}$ is an arc (possibly degenerate) containing $f(p)$, and hence $B_{p q}$ is a subarc (possibly degenerate) of $A_{f(p) f(q)}^{Y}$ containing $f(p)$. Similarly, if $q \in E(T)$ let $C_{p q}=\{f(q)\}$, while if $q \notin E(T)$ let

$$
C_{p q}=\bigcup_{\substack{\left(p^{\prime}, q\right) \in J \\ p^{\prime} \neq p}} A_{f(p) f(q)}^{Y} \cap A_{f\left(p^{\prime}\right) f(q)}^{Y} .
$$

Then, $C_{p q}$ is a subarc (possibly degenerate) of $A_{f(p) f(q)}^{Y}$ containing $f(q)$. From the fact that $T$ is a tree and $\varepsilon$ separates $\Sigma_{T}$, we get that $B_{p q} \cap C_{p q}=\emptyset$. Let $p^{*}$ be the end point of $B_{p q}$ different from $f(p)$ if $B_{p q}$ is nondegenerate or else $p^{*}=f(p)$, and let $q^{*}$ be the end point of $C_{p q}$ different from $f(q)$ if $C_{p q}$ is nondegenerate or else $q^{*}=f(q)$. Note that $B_{p q}=A_{f(p) p^{*}}^{Y}$ and $C_{p q}=A_{q^{*} f(q)}^{Y}$.

Fix $\delta>0$. We want to define a $\delta$-map $g$ from $T$ onto $V$. This is done separately on each $A_{p q}^{X}$ with $(p, q) \in J$ (since $(p, q) \in J$ implies $(q, p) \in J$, we consider only one of the two pairs for every $(p, q) \in J)$. Given $(p, q) \in J$ let $\bar{p}, \bar{q} \in A_{p q}^{X}$ be such that:

- $A_{p \bar{p}}^{X} \cap A_{\bar{q} q}^{X}=\emptyset$,

- $\operatorname{diam}\left(A_{p \bar{p}}^{X}\right)<\frac{\delta}{2}$ and $\operatorname{diam}\left(A_{\bar{q} q}^{X}\right)<\frac{\delta}{2}$,

- $\bar{p}=p$ if and only if $p^{*}=f(p)$,

- $\bar{q}=q$ if and only if $q^{*}=f(q)$.

Let $g$ on $A_{p q}^{X}$ be a homeomorphism onto $A_{f(p) f(q)}^{Y}$ such that $g\left(A_{p \bar{p}}^{X}\right)=B_{p q}$ and $g\left(A_{\bar{q} q}^{X}\right)=C_{p q}$ (and consequently $g(p)=f(p), g(q)=f(q)$ and $\left.g\left(A_{\bar{p} \bar{q}}^{X}\right)=A_{p^{*} q^{*}}^{Y}\right)$. It is straightforward to check that $g$ is a $\delta$-map.

$(4) \Rightarrow(5)$ Fix a tree $T \subseteq X$ and let $V \subseteq Y$ be a tree such that $T \preceq V$. By Theorem 1.7, there is a monotone map $f$ from $V$ onto $T$. Let $r: Y \rightarrow \bar{V}$ be the first point map of $V$. Then $r$ is a monotone map. Hence, $f \circ r$ is a monotone map from $Y$ onto $T$.

$(5) \Rightarrow(2)$ Let $T \subseteq X$ be a tree and fix $\varepsilon>0$. By hypothesis, there is a monotone $\operatorname{map} \varphi$ from $Y$ onto $T$.

Fix a subdivision $\Sigma=(N, E)$ of $T$ such that each element of $E$ has diameter less than $\frac{\varepsilon}{2}$. For each $p \in N$ pick $p^{*} \in \varphi^{-1}(p)$. For every $A=A_{p p^{\prime}}^{T} \in E$, there exists $\psi_{A}: A \rightarrow \varphi^{-1}(A)$ continuous and onto such that $\psi_{A}(p)=p^{*}$ and $\psi_{A}\left(p^{\prime}\right)=p^{\prime *}$. Hence $\psi=\bigcup_{A \in E_{T}} \psi_{A}$ is a function from $T$ onto $Y$. It is straightforward to check that $\psi$ is an $\varepsilon$-map.

Corollary 3.3. Let $X$ and $Y$ be dendrites and let $\left(T_{n}\right)$ and $\left(V_{n}\right)$ be approximating sequences for $X$ and $Y$, respectively. Then

$$
X \preceq Y \Longleftrightarrow \forall n \exists m T_{n} \preceq V_{m} .
$$

Proof. Suppose $X \preceq Y$ and fix $n$. By Theorem 3.2 there exists a subtree $V \subseteq Y$ such that $T_{n} \preceq V$. Let $\varepsilon>0$ be less than the diameter of each edge of $\Sigma_{V}$ and let $m$ be large enough so that every arc in $Y \backslash V_{m}$ has diameter less than $\varepsilon$. Then $V_{m}$ 
contains a homeomorphic copy of $V$. By Lemma 3.1 we have that $V \preceq V_{m}$, and hence $T_{n} \preceq V_{m}$.

For the converse, suppose $\forall n \exists m T_{n} \preceq V_{m}$. Let $T \subseteq X$ be a tree. Let $\varepsilon>0$ be less than the diameter of each edge of $\Sigma_{T}$. There exists $n$ with the property that any arc contained in $X \backslash T_{n}$ has diameter less than $\varepsilon$. Note that $T_{n}$ contains a homeomorphic copy of $T$ and hence by Lemma 3.1, $T \preceq T_{n}$. If $m$ is such that $T_{n} \preceq V_{m}$, we have $T \preceq V_{m}$. Therefore, $X \preceq Y$ by Theorem 3.2

Lemma 3.4. Let $T_{1}$ and $T_{2}$ be trees and $T_{1} \preceq T_{2}$. Then:

(1) $\left|B\left(T_{1}\right)\right| \leq\left|B\left(T_{2}\right)\right|$, and

(2) if $T_{1}$ and $T_{2}$ have the same number of branch points, then $T_{2}$ is the union of a homeomorphic copy of $T_{1}$ and finitely many arcs, each attached to some branch point of this homeomorphic copy.

Proof. This follows from Theorem 1.7

Theorem 3.5. If $D$ is a dendrite such that $B(D)$ is finite, then $\mathcal{Q}(D)=\mathcal{H}(D)$.

Proof. If $D$ is degenerate, then the conclusion is obvious. Hence, let $D$ be nondegenerate and let $C \in \mathcal{Q}(D)$. As $D \preceq C, C$ is a nondegenerate Peano continuum. As $C \preceq D, C$ is a dendrite by Theorem [3.2. Let $T \subseteq C$ be a tree which has as many branch points as $C$. By Theorem [3.2(4), there is a tree $V \subseteq D$ such that $T \preceq V$. By Lemma $3.4(1)$, we have that $|B(T)| \leq|B(V)|$. Hence, $|B(C)| \leq|B(D)|$. A symmetric argument gives us that $|B(C)|=|B(D)|$.

If $k=\max \{\operatorname{ord}(p, D) \mid p \in B(D) \& \operatorname{ord}(p, D)<\infty\}$, let $T \subseteq C$ be a subtree containing $B(C)$ and such that $\operatorname{ord}(q, T)=\operatorname{ord}(q, C)$ when $\operatorname{ord}(q, C)<\infty$, $\operatorname{ord}(q, T)>k$ when $\operatorname{ord}(q, C)$ is infinite. Using Lemma 3.4 (2) and Theorem 3.2(4), we have that $|\{q \in B(C) \mid \operatorname{ord}(q, C)<\infty\}| \leq|\{p \in B(D) \mid \operatorname{ord}(p, D)<\infty\}|$, and by symmetry that these cardinalities are the same.

Another application of Lemma 3.4(2) and Theorem 3.2(4) shows that $C$ and $D$ are homeomorphic.

Definition 3.6. Let the infinite comb $C_{\infty}$ and the infinite $\operatorname{triod} T_{\infty}$ be the following dendrites:

$$
C_{\infty}=(I \times\{0\}) \cup \bigcup_{n \in \mathbb{N}}\left(\left\{\frac{1}{n+2}\right\} \times\left[0, \frac{1}{n+1}\right]\right) \subset I^{2} .
$$

Let $F_{\infty} \subset I^{2}$ be a hairy point, i.e. a dendrite with one branch point $p$ of order $\aleph_{0}$ and end points we denote by $a_{0}, a_{1}, \ldots$ For each $n$ let $b_{n} \in A_{p a_{n}}^{F_{\infty}} \backslash\left\{p, a_{n}\right\}$ and let

$$
T_{\infty}=\left(F_{\infty} \times\{0\}\right) \cup \bigcup_{n \in \mathbb{N}}\left(\left\{b_{n}\right\} \times\left[0, \frac{1}{n+1}\right]\right) \subset I^{3} .
$$

The infinite comb consists of countably many teeth attached on an arc, with length converging to 0 as we approach one of the end points (and nowhere else). The infinite triod is obtained by suitably putting together countably many simple triods pairwise intersecting in the same end point $p$. Homeomorphic copies of $C_{\infty}$ and $T_{\infty}$ are portrayed in Figure 1

Theorem 3.7. $\mathcal{Q}\left(C_{\infty}\right)$ and $\mathcal{Q}\left(T_{\infty}\right)$ are the only two minimal classes under likeness among dendrites with infinitely many branch points. 

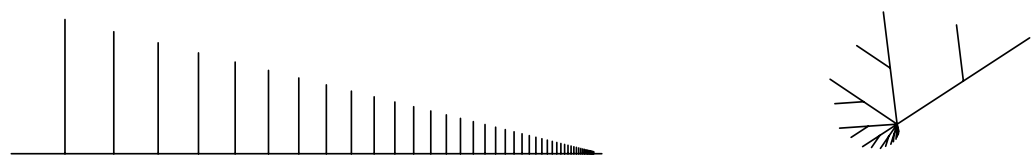

Figure $1 . C_{\infty}$ and $T_{\infty}$

Proof. By Theorem $3.2 T_{\infty} \npreceq C_{\infty}$ and $C_{\infty} \npreceq T_{\infty}$. So it remains to prove that if $D$ is a dendrite with infinitely many branch points, then either $C_{\infty} \preceq D$ or $T_{\infty} \preceq D$.

If there exists a subarc of $D$ containing infinitely many branch points of $D$, then $D$ contains a subcontinuum homeomorphic to $C_{\infty}$ and hence, by Theorem 3.2(2), $C_{\infty} \preceq D$. Otherwise we claim that $D$ contains a subcontinuum homeomorphic to $T_{\infty}$, which yields $T_{\infty} \preceq D$.

To prove the claim note that not all elements of $B(D)$ can have finite order (otherwise a König Lemma style argument yields an arc containing infinitely many of them). Let $p_{0}$ be such that $\operatorname{ord}\left(p_{0}, D\right)=\aleph_{0}$. If $B(D)$ intersects infinitely many connected components of $D \backslash\left\{p_{0}\right\}$ we are done. Otherwise there exists a connected component $Z_{0}$ of $D \backslash\left\{p_{0}\right\}$ containing an infinite subset of $B(D)$. Note that $\overline{Z_{0}}$ is a dendrite and $\overline{Z_{0}}=Z_{0} \cup\left\{p_{0}\right\}$. Repeating the argument, we can find $p_{1} \in Z_{0}$ with $\operatorname{ord}\left(p_{1}, \overline{Z_{0}}\right)=\aleph_{0}$ such that $A_{p_{0} p_{1}}^{D}$ does not contain branching points of infinite order but $p_{0}$ and $p_{1}$. If $B\left(\overline{Z_{0}}\right)$ intersects infinitely many connected components of $\overline{Z_{0}} \backslash\left\{p_{1}\right\}$ we are done; otherwise there exist a connected component $Z_{1}$ of $\overline{Z_{0}} \backslash\left\{p_{1}\right\}$ containing infinitely many points of $B\left(\overline{Z_{0}}\right)$ and a point $p_{2} \in Z_{1}$ of infinite order such that no element of $A_{p_{1} p_{2}}^{D}$ has infinite order except for $p_{1}$ and $p_{2}$. If iterating this procedure we do not find any $n$ such that $B\left(\overline{Z_{n}}\right)$ intersects infinitely many connected components of $\overline{Z_{n}} \backslash\left\{p_{n+1}\right\}$, let $D^{\prime}$ be the smallest subdendrite containing all $p_{n}$ 's. Every branch point of $D^{\prime}$ has finite order in $D^{\prime}$, and we can apply a König Lemma style argument to find an arc containing infinitely many branch points.

Note that we have in fact proved that any dendrite with infinitely many branch points contains a homeomorphic copy of either $C_{\infty}$ or $T_{\infty}$.

Theorem 3.2 implies the following characterizations of the elements of $\mathcal{Q}\left(C_{\infty}\right)$ and $\mathcal{Q}\left(T_{\infty}\right)$.

Corollary 3.8. If $C$ is any continuum, then $C \in \mathcal{Q}\left(C_{\infty}\right)$ if and only if:

(a) $C$ is a dendrite;

(b) $B(C)$ is infinite;

(c) there is an arc $A \subseteq C$ such that $B(C) \subseteq A$.

In other words, dendrites with infinitely many branch points all belonging to a subarc form a single class under quasi-homeomorphism.

Proof. If $C \in \mathcal{Q}\left(C_{\infty}\right)$, then $C$ is a dendrite by the argument used in the proof of Theorem [3.5. By Theorem [3.5 $B(C)$ is infinite. If there is no subarc containing $B(C)$, then $C$ contains a copy of the tree in Figure 2. By Theorem $3.2(4) C \npreceq C_{\infty}$.

If $C$ is such that (a)-(c) hold, then Theorem [3.2(4) easily implies that $C \in$ $\mathcal{Q}\left(C_{\infty}\right)$. 


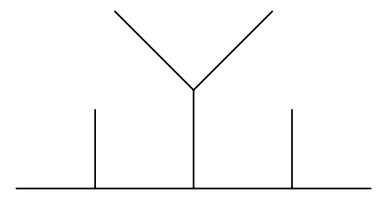

Figure 2. The tree of the proof of Corollary 3.8

Corollary 3.9. If $C$ is any continuum, then $C \in \mathcal{Q}\left(T_{\infty}\right)$ if and only if it is a dendrite that is the union of a dendrite $D$ homeomorphic to $T_{\infty}$ and at most countably many (possibly none) arcs having one end point in the point of order $\aleph_{0}$ of $D$.

In other words, dendrites obtained with infinitely many simple triods and countably many (possibly finitely many or none) arcs glued together at the unique point of order $\aleph_{0}$ form a single class under quasi-homeomorphism.

Proof. If $C \in \mathcal{Q}\left(T_{\infty}\right)$, then Theorem 3.2(4) implies that $|A \cap B(C)| \leq 3$ for any arc $A \subseteq C$. Moreover there exists a unique $p_{0} \in B(C)$ such that $\operatorname{ord}\left(p_{0}, C\right)>3$, and in fact $\operatorname{ord}\left(p_{0}, C\right)=\aleph_{0}$. Since $B(C)$ has to be infinite (otherwise Theorem 3.5 applies), we get the forward implication.

The reverse implication is immediate using Theorem 3.2(4).

Corollaries 3.8 and 3.9 imply that $\mathcal{Q}\left(C_{\infty}\right)$ and $\mathcal{Q}\left(T_{\infty}\right)$ are quasi-homeomorphism classes which are not homeomorphism classes. While it is obvious that $\mathcal{Q}\left(T_{\infty}\right)$ contains only countably many homeomorphism classes (depending on how many arcs appear in the construction described by Corollary [3.9), we will show that $\mathcal{Q}\left(C_{\infty}\right)$ is quite different in this respect (Corollary 6.8).

\section{Combinatorics OF Likeness AMONG DENDRITES}

We are now going to study the quasi-ordering of likeness among dendrites from a combinatorial viewpoint. We start with trees and associate with every tree $T$ a finite partial order with a minimum element (the root) such that the set of predecessors of each element is linearly ordered. In the literature about partial orders such an object is called a finite rooted tree. To this end the finite set is $N_{T}$. To define the partial order we fix $v \in N_{T}$ and let $x \leq y \Longleftrightarrow x \in A_{v y}^{T}$. Let $\tilde{T}=\left(N_{T}, \leq\right)$ and note that $v$ is the root of this partial order.

The definition of $\tilde{T}$ obviously depends on the choice of $v$, but this will not bother us. The first observation is that if $T_{1}$ and $T_{2}$ are trees such that $\tilde{T}_{1}$ and $\tilde{T}_{2}$ are isomorphic as partial orders, then $T_{1}$ and $T_{2}$ are homeomorphic continua. We will obtain a similar implication where the hypothesis is weaker and the (weaker) conclusion is $T_{1} \preceq T_{2}$. The appropriate hypothesis is well known in the theory of partial orders (see e.g. Mil85, p. 490]). In the following definition $x \wedge y$ denotes the greatest lower bound of $x$ and $y$.

Definition 4.1. If $\tilde{T}_{1}$ and $\tilde{T}_{2}$ are finite rooted trees we say that $\tilde{T}_{1}$ is homeomorphically embedded in $\tilde{T}_{2}$, and write $\tilde{T}_{1} \sqsubseteq \tilde{T}_{2}$, if there exists an injective function $f: \tilde{T}_{1} \rightarrow \tilde{T}_{2}$ such that for every $x, y \in \tilde{T}_{1}$ we have $f(x \wedge y)=f(x) \wedge f(y)$.

Lemma 4.2. If $T_{1}$ and $T_{2}$ are trees such that $\tilde{T}_{1} \sqsubseteq \tilde{T}_{2}$, then $T_{2}$ contains a homeomorphic copy of $T_{1}$. 
Proof. Let $f: \tilde{T}_{1} \rightarrow \tilde{T}_{2}$ be a homeomorphic embedding. Let $\leq_{1}$ and $\leq_{2}$ be the partial orders on $\tilde{T}_{1}$ and $\tilde{T}_{2}$ with roots $v_{1}$ and $v_{2}$, respectively. Let $T^{\prime}$ be the smallest subtree of $T_{2}$ containing the range of $f$. Since $f$ is a homeomorphic embedding, $N_{T^{\prime}}$ coincides with the range of $f$. Let $r: T_{2} \rightarrow T^{\prime}$ be the first point map for $T^{\prime}$. If $f\left(v_{1}\right) \neq r\left(v_{2}\right)$ there exists $u \in N_{T_{1}}$ with $r\left(v_{2}\right) \in A_{f\left(v_{1}\right) f(u)}^{T_{2}}$, against $f\left(v_{1}\right) \leq_{2} f(u)$. Therefore $f\left(v_{1}\right)=r\left(v_{2}\right)$.

Define the partial order $\leq^{\prime}$ on $N_{T^{\prime}}$ so that $f\left(v_{1}\right)$ (which belongs to $N_{T^{\prime}}$ ) is the root. Again using the fact that $f$ is a homeomorphic embedding, we have $x \leq_{1} y \Longleftrightarrow f(x) \leq_{2} f(y) \Longleftrightarrow f(x) \leq^{\prime} f(y)$. Therefore

$$
\begin{aligned}
A_{x y}^{T_{1}} \in E_{T_{1}} & \Longleftrightarrow \text { no element of } N_{T_{1}} \text { is between } x \text { and } y \text { in } \leq_{1} \\
& \Longleftrightarrow \text { no element of } N_{T^{\prime}} \text { is between } f(x) \text { and } f(y) \text { in } \leq^{\prime} \\
& \Longleftrightarrow A_{f(x) f(y)}^{T^{\prime}} \in E_{T^{\prime}} .
\end{aligned}
$$

This equivalence shows that $T^{\prime}$ and $T_{1}$ are homeomorphic.

Corollary 4.3. If $T_{1}$ and $T_{2}$ are trees such that $\tilde{T}_{1} \sqsubseteq \tilde{T}_{2}$, then $T_{1} \preceq T_{2}$.

Proof. By Theorem 1.7 and Lemma 4.2.

The relation $\sqsubseteq$ of homeomorphic embedding is a quasi-ordering on the class of finite rooted trees. As such it has been extensively studied: Kruskal proved that it is a well quasi-ordering (wqo), i.e. that it is well-founded and all sets of pairwise incomparable elements (antichains) are finite. Laver improved this result by showing that it is indeed a better quasi-ordering (bqo). Here we give a descriptive set-theoretic definition of bqo, due to Simpson ([Sim85]). A survey of wqo and bqo theory is Mil85.

Definition 4.4. Let $(Q, \leq)$ be a quasi-ordering. Let $[\mathbb{N}]^{\mathbb{N}}$ be the set of all infinite subsets of $\mathbb{N}$, with the topology inherited from the Baire space $\mathbb{N}^{\mathbb{N}} .(Q, \leq)$ is a $b q o$ if, endowing $Q$ with the discrete topology, for every continuous function $f:[\mathbb{N}]^{\mathbb{N}} \rightarrow Q$ there exists $X \in[\mathbb{N}]^{\mathbb{N}}$ such that $f(X) \leq f(X \backslash\{\min X\})$.

It is immediate that if $\left(Q, \leq_{Q}\right)$ and $\left(Q^{\prime}, \leq_{Q^{\prime}}\right)$ are quasi-ordering, the latter is a bqo and there exists $f: Q \rightarrow Q^{\prime}$ such that $f(x) \leq_{Q^{\prime}} f(y)$ implies $x \leq_{Q} y$, then $\left(Q, \leq_{Q}\right)$ is also a bqo. This observation is used in the proofs of the following corollaries.

Corollary 4.5. The relation $\preceq$ is bqo on trees.

Proof. This follows immediately from Laver's result and Corollary 4.3

Corollary 3.3 shows that $\preceq$ on dendrites is understood by looking at $\preceq$ on infinite sequences of trees. It can be argued that bqo theory was born precisely to be able to deal with this sort of infinitary operations.

Theorem 4.6. The relation $\preceq$ is bqo on dendrites.

Proof. Define the following relation between infinite sequences of trees:

$$
\left(T_{i}\right) \preceq^{\prime}\left(T_{i}^{\prime}\right) \Longleftrightarrow \forall n \exists m T_{n} \preceq T_{m}^{\prime} .
$$

Since $\preceq$ is bqo on trees, $\preceq^{\prime}$ is also bqo by a theorem of Nash-Williams' (see e.g. [Sim85, Corollary 9.20] or [Mil85 Theorem 2.22]).

By Corollary 3.3 if $\left(T_{n}\right)$ and $\left(V_{n}\right)$ are approximating sequences for dendrites $X$ and $Y$, then $\left(T_{n}\right) \preceq^{\prime}\left(V_{n}\right)$ implies $X \preceq Y$. 
In particular Theorem 4.6 implies that $\preceq$ on dendrites is well-founded and has no infinite antichains.

Corollary 4.5 allows us to compute the number of quasi-homeomorphism classes in which dendrites are partitioned.

Theorem 4.7. There are countably many quasi-homeomorphism classes among dendrites.

Proof. It is obvious that there are countably many quasi-homeomorphism classes of trees. By Corollary 4.5 these are bqo under $\preceq$ and by a theorem of Laver (Lav71, Theorem 4.11]) there are countably many classes of infinite sequences of trees under the equivalence relation induced by $\preceq^{\prime}$ (the relation defined in the proof of Theorem 4.6). By Corollary 3.3 we obtain our statement.

\section{The COMPlexity of Being $\mathcal{C}$-Like}

Before we can proceed to study the homeomorphism relation on dendrites, we need to establish some facts about the complexity of the family of $\mathcal{C}$-like continua, where $\mathcal{C}$ is any nonempty class of continua whose members satisfy a property we are going to describe in Lemma 5.2. Besides being used in all subsequent sections, these results are interesting on their own. They also allow the use of Baire category arguments to prove some folklore facts in continuum theory.

Definition 5.1. A separable metric space $X$ is an absolute retract if whenever $X$ is embedded as a closed subset of a separable metric space $Y$, there exists a continuous function $f: Y \rightarrow X$ which is the identity on $X$.

The following two facts will be used in the proof of the next lemma.

The Borsuk Extension Theorem ([vM89, Theorem 1.5.2]) asserts that if $X$ is an absolute retract, $C$ is a closed subset of the separable metric space $Y$ and $f: C \rightarrow X$ is continuous, then there exists a continuous function $f^{\prime}: Y \rightarrow X$ which extends $f$.

Suppose that $F_{1}, F_{2}, \ldots, F_{n}$ are closed subsets of a compact metric space $X$. Then there exist open sets $U_{1}, \ldots, U_{n}$ with $U_{i} \supseteq F_{i}$ such that for any set $J \subseteq$ $\{1,2, \ldots, n\}, \bigcap_{i \in J} F_{i} \neq \emptyset$ if and only if $\bigcap_{i \in J} \overline{U_{i}} \neq \emptyset$.

Lemma 5.2. Let $C$ be a continuum. Suppose that for each $\delta>0$ there exist finitely many subcontinua $A_{1}, \ldots, A_{m} \subseteq C$ such that the following hold:

(1) $\bigoplus_{i=1}^{m} A_{i}=C$;

(2) $\operatorname{diam}\left(A_{i}\right)<\delta$ for each $i=1, \ldots, m$;

(3) for each $J \subseteq\{1, \ldots, m\}$, if $\bigcap_{i \in J} A_{i} \neq \emptyset$, then $\bigcap_{i \in J} A_{i}$ is an absolute retract (in particular, each $A_{i}$ is an absolute retract).

Then, for every $\varepsilon>0$ the set

$$
\mathcal{U}=\left\{M \in \mathrm{C}\left(I^{\mathbb{N}}\right) \mid \text { there is an } \varepsilon \text {-map from } M \text { onto } C\right\}
$$

is open in $\mathrm{C}\left(I^{\mathbb{N}}\right)$.

Proof. When $C$ is degenerate we have that $\mathcal{U}$ is $\left\{M \in \mathrm{C}\left(I^{\mathbb{N}}\right) \mid \operatorname{diam}(M)<\varepsilon\right\}$, which is open. Therefore, we may assume that $C$ is nondegenerate. Let $M \in \mathcal{U}$ and fix an $\varepsilon$-map $f$ from $M$ onto $C$. Since $f$ is an $\varepsilon$-map there exists $\eta$ such that for all $B \subseteq C$ with $\operatorname{diam}(B)<\eta$ we have $\operatorname{diam}\left(f^{-1}(B)\right)<\varepsilon$. Applying the hypothesis on $C$ to $\delta=\frac{\eta}{2}$, there exist subcontinua $A_{1}, \ldots, A_{m} \subseteq C$ (we may assume $m \geq 2$ ) such that (1)-(3) above hold. Observe that our choice of $\delta$ implies that 
(4) if $J \subseteq\{1, \ldots, m\}$ and $\bigcap_{i \in J} A_{i} \neq \emptyset$, then $\operatorname{diam}\left(f^{-1}\left(\bigcup_{i \in J} A_{i}\right)\right)<\varepsilon$.

Let $\mathcal{J}$ be the collection of all $J \subseteq\{1, \ldots, m\}$ such that $\bigcap_{i \in J} A_{i} \neq \emptyset$. Since $C=$ $\bigoplus_{i=1}^{m} A_{i}$, we may choose nonempty open sets $O_{i} \subseteq A_{i}, 1 \leq i \leq m$, such that $\overline{O_{j}} \cap A_{i}=\emptyset$ for all $j \neq i$. Note that this implies that sufficiently small open sets containing $f^{-1}\left(A_{i}\right), 1 \leq i \leq m$, separate $f^{-1}\left(A_{i}\right)$ and $f^{-1}\left(\overline{O_{j}}\right)$ for $i \neq j$. Using the remark before the lemma, this fact, and (4), we may obtain a sequence of open sets (in $I^{\mathbb{N}}$ ) $V_{1}, \ldots, V_{m}$ such that:

(i) for all $1 \leq i \leq m, f^{-1}\left(A_{i}\right) \subseteq V_{i}$,

(ii) for all $J \in \mathcal{J}, \operatorname{diam}\left(\bigcup_{i \in J} \overline{V_{i}}\right)<\varepsilon$,

(iii) if $J \subseteq\{1, \ldots, m\}$, then $\bigcap_{i \in J} \overline{V_{i}} \neq \emptyset$ if and only if $J \in \mathcal{J}$, and

(iv) if $U_{i}=V_{i} \backslash\left(\bigcup_{j \neq i} \overline{V_{j}}\right)$, then $U_{i} \cap f^{-1}\left(A_{i}\right) \neq \emptyset$.

Let $V^{J}=\bigcap_{i \in J} \overline{V_{i}}$ and $A^{J}=\bigcap_{i \in J} A_{i}$ for $J \in \mathcal{J}$.

We will show that the open set

$$
\mathcal{O}=\left\{N \in \mathrm{C}\left(I^{\mathbb{N}}\right) \mid N \subseteq \bigcup_{i=1}^{n} V_{i} \& \forall i N \cap U_{i} \neq \emptyset\right\}
$$

is included in $\mathcal{U}$. Since $M \in \mathcal{O}$ this proves that $\mathcal{U}$ is open.

Let $N \in \mathcal{O}$ and note that, since $m \geq 2, N$ is nondegenerate. We will define by steps an $\varepsilon$-map $g: \bigcup_{i=1}^{n} \overline{V_{i}} \rightarrow C$ such that $g(N)=C$, thereby showing that $N \in \mathcal{U}$.

To begin with, by the Boundary Bumping Theorem ([Nad92, Corollary 5.5]), for each $i$ there exists a nondegenerate continuum $N_{i} \subseteq N \cap U_{i}$. Since the $U_{i}$ 's are pairwise disjoint, so are the $N_{i}$ 's. Since absolute retracts are locally connected ( vM89 exercise 1.5.3]), every $A_{i}$ is a Peano continuum and hence the continuous image of any nondegenerate continuum. We can thus define $g$ on $N_{i}$ so that $g\left(N_{i}\right)=$ $A_{i}$. Note that at this first stage we already made sure that $g(N)=C$.

The extension of $g$ to a continuous function on all of $\bigcup_{i=1}^{m} \overline{V_{i}}$ is done by induction. For each $1 \leq i \leq m$, let $\mathcal{J}_{i}$ be the set of $J \in \mathcal{J}$ such that $|J|=i$. Let $k$ be the largest integer such that $\mathcal{J}_{k}$ is nonempty. Note that $\mathcal{J}_{\ell}$ is nonempty for all $1 \leq \ell \leq k$ and that $k>1$ since $m>1$. For each $J \in \mathcal{J}_{k}$, let $g$ be a continuous function from $V^{J}$ into $A^{J}$. We note that $g$ is well defined at this stage because if $J, J^{\prime} \in \mathcal{J}_{k}$ and $J \neq J^{\prime}$, then $V^{J} \cap V^{J^{\prime}}=\emptyset$ by our choice of $k$ and (iii). At stage $k-1$ for every $J \in \mathcal{J}_{k-1}$ define $g$ on $V^{J}$ so that $g$ is an extension of the existing $g$ defined at stage $k$ and $f\left(V^{J}\right) \subseteq A^{J}$. This is possible by the Borsuk Extension Theorem and (iii). Notice that if $J \neq J^{\prime}$ with both in $\mathcal{J}_{k-1}$ and $V^{J} \cap V^{J^{\prime}} \neq \emptyset$, then by (iii) $J \cup J^{\prime} \in \mathcal{J}_{k}$ and $g$ has already been defined on $V^{J \cup J^{\prime}}=V^{J} \cap V^{J^{\prime}}$ at stage $k$. Hence, $g$ is well defined at stage $k-1$. Repeating this process we reach the first stage without conflicts with the definition of $g$ on the $N_{i}$ 's. At the first stage, we make sure the definition of $g$ on the $\overline{V_{i}}$ 's agrees with the definition of $g$ on the previous $V^{J}$ 's as well as the $N_{i}$ 's. The resulting function $g$ is well defined on all of $\bigcup_{i=1}^{m} \overline{V_{i}}$.

Finally, let us see that $g$ is indeed an $\varepsilon$-map. Let $p \in C$. Let $J$ be the set of all $i$ 's such that $p \in A_{i}$, so that $J \in \mathcal{J}$. By construction, we have that $g^{-1}(p) \subseteq \bigcup_{i \in J} \bar{V}_{i}$. By (ii), we have that $\operatorname{diam}\left(g^{-1}(p)\right)<\varepsilon$.

There are various classes of continua satisfying the hypotheses of Lemma 5.2 for example, dendrites, finitely triangulable continua (a topological space is finitely triangulable if it is homeomorphic to the geometric realization of a finite simplicial complex, and these spaces include $n$-cells and $n$-spheres) and the Hilbert cube $I^{\mathbb{N}}$. 
Theorem 5.3. Let $\mathcal{C}$ be a nonempty class of continua satisfying the hypothesis of Lemma [5.2. The set $\mathcal{L}_{\mathcal{C}}$ of $\mathcal{C}$-like continua is $\boldsymbol{\Pi}_{2}^{0}$. Moreover if $\mathcal{C}$ contains at least one nondegenerate continuum and there exists a nondegenerate continuum which does not belong to $\mathcal{L}_{\mathcal{C}}$, then $\mathcal{L}_{\mathcal{C}}$ is $\boldsymbol{\Pi}_{2}^{0}$-complete.

Proof. The first assertion is immediate from the definition of $\mathcal{C}$-like continuum and Lemma5.2. The second assertion follows from the Baire Category Theorem because a dense set with dense complement cannot be both $\boldsymbol{\Pi}_{2}^{0}$ and $\boldsymbol{\Sigma}_{2}^{0}$.

Corollary 5.4. (1) If $C$ is a nondegenerate finitely triangulable continuum or a nondegenerate dendrite, then $\mathcal{L}_{C}$ is $\boldsymbol{\Pi}_{2}^{0}$-complete;

(2) the set of graph-like continua is $\boldsymbol{\Pi}_{2}^{0}$-complete;

(3) the set of tree-like continua is $\boldsymbol{\Pi}_{2}^{0}$-complete;

(4) more in general, for any class $\mathcal{C}$ of continua of bounded dimension satisfying the hypothesis of Lemma 5.2 and containing a nondegenerate continuum, $\mathcal{L}_{\mathcal{C}}$ is $\Pi_{2}^{0}$-complete.

Proof. This follows immediately from Theorem [5.3. To prove (4) we use the fact that if every continuum in $\mathcal{C}$ has dimension $\leq n$, then every $\mathcal{C}$-like continuum has dimension $\leq n$ (see Ale28]).

We next use the Baire Category Theorem to prove some folklore facts in continuum theory. Recall that a pseudoarc is a hereditarily indecomposable arc-like continuum and a pseudocircle is a planar circle-like hereditarily indecomposable continuum which separates the plane. Also, recall that pseudoarcs and pseudocircles are unique up to homeomorphism.

Corollary 5.5. Let $G$ be a nondegenerate finitely triangulable continuum. Then, the pseudoarc is $G$-like. More generally this holds if $G$ is a nondegenerate continuum satisfying the hypotheses of Lemma[5.2.

Proof. The collection $\mathcal{P}$ of pseudoarcs is comeager in $\mathrm{C}\left(I^{\mathbb{N}}\right)$ ([Bin51]). $\mathcal{L}_{G}$ is dense and $G_{\delta}$ by Corollary 5.4 and hence comeager. By the Baire Category Theorem $\mathcal{P} \cap \mathcal{L}_{G} \neq \emptyset$ and hence the pseudoarc is $G$-like.

Corollary 5.6. Let $G$ be a graph which contains a homeomorphic copy of $S^{1}$. Then, the pseudocircle is G-like.

Proof. Let $A$ be a closed annulus in the plane which separates the origin from $(1,1)$. Let $\mathcal{M}$ be the collection of all subcontinua of $A$ which separate the origin from $(1,1)$. Then, $\mathcal{M}$ is a closed subset of $\mathrm{C}(A)$ and hence a compact set itself.

Let $\mathcal{H}_{1}$ be the collection of those continua in $\mathcal{M}$ which are $G$-like. By Corollary 5.4, we know that $\mathcal{H}_{1}$ is $G_{\delta}$. By a slight modification of the argument that the collection of arcs is dense in $\mathrm{C}(A)$, we can obtain that the set of continua in $\mathcal{M}$ homeomorphic to $G$ is dense in $\mathcal{M}$. Hence, $\mathcal{H}_{1}$ is comeager in $\mathcal{M}$. Similarly, $\mathcal{H}_{2}$, the collection of those continua in $\mathcal{M}$ which are circle-like, is comeager in $\mathcal{M}$.

Recall that the collection of hereditarily indecomposable continua is a $G_{\delta}$ subset of $\mathrm{C}\left(I^{\mathbb{N}}\right)$. Using the fact that the collection of continua in $\mathcal{M}$ homeomorphic to $S^{1}$ is dense in $\mathcal{M}$, we can approximate any continuum in $\mathcal{M}$ by a pseudocircle in $\mathcal{M}$. Hence, $\mathcal{H}_{3}$, the collection of hereditarily indecomposable continua in $\mathcal{M}$, is comeager in $\mathcal{M}$.

Now, let $M \in \mathcal{H}_{1} \cap \mathcal{H}_{2} \cap \mathcal{H}_{3}$. Since $M \in \mathcal{H}_{2} \cap \mathcal{H}_{3}, M$ is a pseudocircle. Since $M \in \mathcal{H}_{1}, M$ is $G$-like. 


\section{Complexity of the homeOmorphism Relation}

For some classes of continua (like dendrites with a finite number of branch points; see Theorem 3.5) the homeomorphism and the quasi-homeomorphism classes coincide. This will allow us to find the complexity of some homeomorphism class of continua computing in fact the complexity of the class of quasi-homeomorphism (Theorems 8.5 and 8.7). Corollaries 3.8 and 3.9 show that this argument fails when one considers the infinite triod and the infinite comb, which are the minimal dendrites with infinitely many branch points (Theorem 3.7).

Despite their similar position with respect to the quasi-ordering of likeness, the structure of homeomorphism on $\mathcal{Q}\left(C_{\infty}\right)$ and $\mathcal{Q}\left(T_{\infty}\right)$ is very different. Indeed, as already noted in Section $3, \mathcal{Q}\left(T_{\infty}\right)$ is partitioned in countably many homeomorphism classes (in particular, homeomorphism on $\mathcal{Q}\left(T_{\infty}\right)$ is Borel reducible to equality on $\mathbb{N})$. In contrast, we will now show that the quotient of $\mathcal{Q}\left(C_{\infty}\right)$ under homeomorphism has very high definable cardinality, namely the relation of homeomorphism on $\mathcal{Q}\left(C_{\infty}\right)$ is $S_{\infty}$-universal. In doing this we show that homeomorphism on the class of all dendrites is $S_{\infty}$-universal as well.

Though later we shall study in more detail the descriptive complexity of some classes of continua, we already need to prove here two such results (later it will be shown that these computations are sharp).

Lemma 6.1. The classes of Peano continua and of dendrites are $\boldsymbol{\Pi}_{3}^{0}$.

Proof. Let $X \in \mathrm{C}\left(I^{\mathbb{N}}\right)$. Then (see $\mathrm{Nad}$ 2, Theorem 8.4]) $X$ is Peano if and only if for each $\varepsilon>0, X$ is the union of finitely many subcontinua of diameter less than $\varepsilon$. This means

$$
\forall \varepsilon>0 \exists n \exists\left(X_{0}, \ldots, X_{n}\right) \in \mathrm{C}\left(I^{\mathbb{N}}\right)^{n+1}\left(\forall i \leq n \operatorname{diam}\left(X_{i}\right) \leq \varepsilon \& \bigcup_{i=0}^{n} X_{i}=X\right) .
$$

Therefore the class of Peano continua is $\boldsymbol{\Pi}_{3}^{0}$.

Dendrites are exactly the Peano continua which are tree-like (see Nad92, exercise 10.50]). The computation just completed and Corollary 5.4 imply that the class of dendrites is $\boldsymbol{\Pi}_{3}^{0}$.

From now on let $\mathcal{P} \subset \mathrm{C}\left(I^{\mathbb{N}}\right)$ be the class of Peano continua and $\mathcal{D} \subset \mathrm{C}\left(I^{\mathbb{N}}\right)$ be the class of dendrites.

Lemma 6.2. If $D$ is a nondegenerate dendrite, then the class $\mathcal{Q}(D)$ is $\Pi_{3}^{0}$.

Proof. By Theorem 4.7 there are only countably many quasi-homeomorphism classes containing dendrites; thus there exists a countable set of dendrites $\left\{D_{n} \mid\right.$ $n \in \mathbb{N}\}$ such that $\forall n D_{n} \prec D$ and $\forall D^{\prime} \prec D \exists n \mathcal{Q}\left(D^{\prime}\right)=\mathcal{Q}\left(D_{n}\right)$. Since it is obvious that

$$
\mathcal{Q}(D)=\left(\mathcal{P} \cap \mathcal{L}_{D}\right) \backslash \bigcup_{n \in \mathbb{N}} \mathcal{L}_{D_{n}}
$$

by Lemma 6.1 and Corollary $5.4 \mathcal{Q}(D)$ is $\boldsymbol{\Pi}_{3}^{0}$.

Definition 6.3. Let

$$
\begin{aligned}
& \mathcal{B}^{\mathcal{D}}=\left\{(X, x) \in \mathrm{C}\left(I^{\mathbb{N}}\right) \times I^{\mathbb{N}} \mid X \in \mathcal{D} \& x \in B(X)\right\} \\
& \mathcal{E}^{\mathcal{D}}=\left\{(X, x) \in \mathrm{C}\left(I^{\mathbb{N}}\right) \times I^{\mathbb{N}} \mid X \in \mathcal{D} \& x \in E(X)\right\} .
\end{aligned}
$$


Lemma 6.4. $\mathcal{B}^{\mathcal{D}}$ and $\mathcal{E}^{\mathcal{D}}$ are Borel subsets of $\mathrm{C}\left(I^{\mathbb{N}}\right) \times I^{\mathbb{N}}$.

Proof. Let $X \in \mathrm{C}\left(I^{\mathbb{N}}\right)$ and $x \in I^{\mathbb{N}}$.

We have that $(X, x) \in \mathcal{B}^{\mathcal{D}}$ if and only if $x \in X, X \in \mathcal{D}$ and there exist $x_{1}, x_{2}, x_{3} \in$ $X \backslash\{x\}$ such that for every $L \in \mathrm{C}\left(I^{\mathbb{N}}\right)$ with $L \subseteq X$ we have

$\left(x_{1} \in L \& x_{2} \in L\right) \vee\left(x_{1} \in L \& x_{3} \in L\right) \vee\left(x_{2} \in L \& x_{3} \in L\right) \Longrightarrow x \in L$.

Therefore $\mathcal{B}^{\mathcal{D}}$ is $\boldsymbol{\Sigma}_{1}^{1}$. On the other hand, $(X, x) \in \mathcal{B}^{\mathcal{D}}$ if and only if $x \in X, X \in \mathcal{D}$ and there exists $\varepsilon>0$ such that for every $K \in \mathrm{K}\left(I^{\mathbb{N}}\right)$ with $x \notin K$ and $K \subseteq X$ we have

$$
\forall y \in I^{\mathbb{N}}(y \in X \& d(y, x) \geq \varepsilon \Longrightarrow y \in K) \Longrightarrow|K \cap \overline{X \backslash K}|>2 .
$$

Since the maps $\mathrm{K}\left(I^{\mathbb{N}}\right)^{2} \rightarrow \mathrm{K}\left(I^{\mathbb{N}}\right),\left(L, L^{\prime}\right) \mapsto \overline{L \backslash L^{\prime}}$ and $\left(L, L^{\prime}\right) \mapsto L \cap L^{\prime}$ are Borel (see [Kur68]), and the compact sets of cardinality greater than 2 form a Borel subset of $\mathrm{K}\left(I^{\mathbb{N}}\right)$, this shows that $\mathcal{B}^{\mathcal{D}}$ is also $\Pi_{1}^{1}$. By Souslin's Theorem, $\mathcal{B}^{\mathcal{D}}$ is Borel.

To see that $\mathcal{E}^{\mathcal{D}}$ is $\boldsymbol{\Sigma}_{1}^{1}$, note that $(X, x) \in \mathcal{E}^{\mathcal{D}}$ if and only if $x \in X, X \in \mathcal{D}$ and for all $\varepsilon>0$ there exists $K \in \mathrm{K}\left(I^{\mathbb{N}}\right)$ with $x \notin K$ and $K \subseteq X$ such that

$$
\forall y \in I^{\mathbb{N}}(y \in X \& d(x, y) \geq \varepsilon \Longrightarrow y \in K) \&|K \cap \overline{X \backslash K}|=1 .
$$

On the other hand, $(X, x) \in \mathcal{E}^{\mathcal{D}}$ if and only if $x \in X, X \in \mathcal{D}$ and for every $x_{1}, x_{2} \in X \backslash\{x\}$ we have

$$
\exists L \in \mathrm{C}\left(I^{\mathbb{N}}\right)\left(x_{1}, x_{2} \in L \& L \subseteq X \& x \notin L\right) .
$$

Therefore $\mathcal{E}^{\mathcal{D}}$ is $\Pi_{1}^{1}$ too, and hence Borel.

Lemma 6.5. The homeomorphism relation on dendrites is classifiable by countable structures.

Proof. Let $\mathcal{D}_{\infty} \subseteq \mathrm{C}\left(I^{\mathbb{N}}\right)$ be the class of dendrites in $I^{\mathbb{N}}$ having infinitely many branch points. Since there are countably many homeomorphism classes of dendrites with finitely many branch points, by Lemma 6.1 and Theorem $1.3, \mathcal{D}_{\infty}$ is Borel. Moreover, adding countably many Borel classes does not harm classifiability by countable structures of an equivalence relation, so it is enough to prove that the homeomorphism relation on $\mathcal{D}_{\infty}$ is classifiable by countable structures.

Let $\mathcal{B}^{\infty}=\mathcal{B}^{\mathcal{D}} \cap\left(\mathcal{D}_{\infty} \times I^{\mathbb{N}}\right)$. By Lemma 6.4 $\mathcal{B}^{\infty}$ is a Borel subset of $\mathrm{C}\left(I^{\mathbb{N}}\right) \times I^{\mathbb{N}}$. Moreover, for every $X \in \mathcal{D}_{\infty}$, the section $\left\{x \in I^{\mathbb{N}} \mid(X, x) \in \mathcal{B}^{\infty}\right\}$ is countably infinite. By a consequence of Lusin-Novikov Uniformization Theorem (see Kec95. Exercise 18.15]), there exist a sequence of Borel functions $\mathcal{D}_{\infty} \rightarrow I^{\mathbb{N}},\left(b_{n}\right)$ such that:

- $\left\{x \in I^{\mathbb{N}} \mid(X, x) \in \mathcal{B}\right\}=\left\{b_{n}(X) \mid n \in \mathbb{N}\right\}$ for all $X \in \mathcal{D}_{\infty}$;

- $b_{n}(X) \neq b_{m}(X)$ for all $X \in \mathcal{D}_{\infty}$ and $n \neq m$.

Branch points (and their relative position) do not encode all the information needed to characterize the homeomorphism class of $X \in \mathcal{D}_{\infty}$ : to this end we also need to have information about maximal open free $\operatorname{arcs}$ of $X$.

Let $\mathcal{T} \subseteq \mathrm{C}\left(I^{\mathbb{N}}\right)^{2}$ be the set of all pairs $(X, A)$ such that $X \in \mathcal{D}_{\infty}$ and $A$ is the closure of a maximal open free $\operatorname{arc}$ of $X$. We want to show that $\mathcal{T}$ is Borel. To this end note that a subarc $A$ of $X \in \mathcal{D}_{\infty}$ is the closure of a maximal open free arc of $X$ if and only if $A$ does not contain any branch points of $X$ except possibly as end points and either

- one end point of $A$ is an end point of $X$ while the other end point of $A$ belongs to $\overline{B(X)}$, or

- both end points of $A$ belong to $\overline{B(X)}$. 
Let $X, A \in \mathrm{C}\left(I^{\mathbb{N}}\right)$. Then $(X, A) \in \mathcal{T}$ if and only if the following properties hold:

- $A \subseteq X, A$ is an arc and $X \in \mathcal{D}_{\infty}$;

- $\left(A, b_{n}(X)\right) \in \mathcal{E}^{\mathcal{D}}$ whenever $b_{n}(X) \in A$;

- either

- $\overline{X \backslash A} \in \mathrm{C}\left(I^{\mathbb{N}}\right)$ and $\forall \varepsilon>0 \exists n d\left(b_{n}(X), A\right)<\varepsilon$, or

- there exists $\delta>0$ such that for all $\varepsilon>0$ there exist $n$ and $m$ satisfying $d\left(b_{n}(X), b_{m}(X)\right)>\delta, d\left(b_{n}(X), A\right)<\varepsilon$ and $d\left(b_{m}(X), A\right)<\varepsilon$.

By Lemma 6.4 $\mathcal{T}$ is indeed Borel.

For every $X \in \mathcal{D}_{\infty}$ the maximal open free arcs of $X$ are pairwise disjoint and hence each nonempty section of $\mathcal{T}$ is countable. For $0 \leq i \leq \aleph_{0}$ let

$$
\mathcal{D}_{\infty}^{i}=\left\{X \in \mathcal{D}_{\infty}||\left\{A \in \mathrm{C}\left(I^{\mathbb{N}}\right) \mid(X, A) \in \mathcal{T}\right\} \mid=i\right\}
$$

be the set of all the elements of $\mathcal{D}_{\infty}$ having exactly $i$ maximal open free arcs. Again using [Kec95, Exercise 18.15], $\mathcal{D}_{\infty}^{i}$ is Borel for each $i$ and there exist sequences $\left(h_{n}^{i}\right.$ : $n<i$ ) of Borel functions $\mathcal{D}_{\infty}^{i} \rightarrow \mathrm{C}\left(I^{\mathbb{N}}\right)$ that injectively enumerate the nonempty sections of $\mathcal{T}$.

Note that each $\mathcal{D}_{\infty}^{i}$ is closed under homeomorphism. Moreover, the disjoint sum of a countable collection of equivalence relations classifiable by countable structures is still classifiable by countable structures. So, to conclude the proof, it will be enough to show that, for $0 \leq i \leq \aleph_{0}$, the homeomorphism relation on $\mathcal{D}_{\infty}^{i}$ is classifiable by countable structures. To this aim we shall define a countable firstorder language $\mathcal{L}$ and a Borel function $\Phi_{i}: \mathcal{D}_{\infty}^{i} \rightarrow X_{\mathcal{L}}$ such that, for all $X, X^{\prime} \in \mathcal{D}_{\infty}^{i}$, $X$ and $X^{\prime}$ are homeomorphic if and only if $\Phi_{i}(X)$ and $\Phi_{i}\left(X^{\prime}\right)$ are isomorphic. We will explicitly do this for $i=\aleph_{0}$ and leave the other similar cases to the reader. To simplify the notation we will use $\Phi$ and $h_{n}$ instead of $\Phi_{\aleph_{0}}$ and $h_{n}^{\aleph_{0}}$.

We need one more ingredient for describing the construction of the structures $\Phi(X)$. Let $\mathcal{A}$ be the set of all $\left(X, K, K^{\prime}, K^{\prime \prime}\right) \in \mathrm{C}\left(I^{\mathbb{N}}\right)^{4}$ such that

$$
X \in \mathcal{D} \& K, K^{\prime}, K^{\prime \prime} \subseteq X \& \forall x \in K \forall x^{\prime} \in K^{\prime} \forall x^{\prime \prime} \in K^{\prime \prime} x^{\prime} \in A_{x x^{\prime \prime}}^{X} .
$$

To see that $\mathcal{A}$ is Borel note that in this formula $x^{\prime} \in A_{x x^{\prime \prime}}^{X}$ can be replaced by

$$
\forall L \in \mathrm{C}\left(I^{\mathbb{N}}\right)\left(L \subseteq X \& x \in L \& x^{\prime \prime} \in L \Longrightarrow x^{\prime} \in L\right) .
$$

Let $\mathcal{L}=\{P, R\}$ be the language with $P$ a unary relation symbol and $R$ a ternary relation symbol (actually $P$ could be dispensed with, since $P$ will be definable from $R$ within each $\Phi(X))$. If $M$ is a $\mathcal{L}$-structure on $\mathbb{N}$, denote by $P^{M}$ and $R^{M}$ the interpretations of $P$ and $R$ in $M$, so that $P^{M} \subseteq \mathbb{N}$ and $R^{M} \subseteq \mathbb{N}^{3}$.

Given $X \in \mathcal{D}_{\infty}^{\aleph_{0}}$, we build an $\mathcal{L}$-structure $\Phi(X)$ whose universe is essentially $\left\{\left\{b_{n}(X)\right\} \mid n \in \mathbb{N}\right\} \cup\left\{h_{n}(X) \mid n \in \mathbb{N}\right\}$, with $P$ identifying $\left\{\left\{b_{n}(X)\right\} \mid n \in \mathbb{N}\right\}$ and $R$ describing how the continua are mutually located within $X$. To precisely define $\Phi(X)$ we need to specify $P^{\Phi(X)}$ and $R^{\Phi(X)}$. To ease the description of $\Phi(X)$, let $q_{2 n}(X)=\left\{b_{n}(X)\right\}$ and $q_{2 n+1}(X)=h_{n}(X)$ for each $n$.

Given $X \in \mathcal{D}_{\infty}^{\aleph_{0}}$, let

- $P^{\Phi(X)}=\{m \mid m$ is even $\}$;

- $R^{\Phi(X)}=\left\{\left(m, m^{\prime}, m^{\prime \prime}\right) \mid\left(X, q_{m}(X), q_{m^{\prime}}(X), q_{m^{\prime \prime}}(X)\right) \in \mathcal{A}\right\}$.

$\Phi(X)$ is an $\mathcal{L}$-structure and, since the $q_{m}$ 's and $\mathcal{A}$ are Borel, the function $\Phi$ is Borel. We now want to show that $X$ and $X^{\prime}$ are homeomorphic continua if and only if $\Phi(X)$ and $\Phi\left(X^{\prime}\right)$ are isomorphic $\mathcal{L}$-structures, for every $X, X^{\prime} \in \mathcal{D}_{\infty}^{\aleph_{0}}$. 
Let $X$ and $X^{\prime}$ be homeomorphic via a homeomorphism $\varphi: X \rightarrow X^{\prime}$. Define $\psi: \mathbb{N} \rightarrow \mathbb{N}$ by

- $\psi(2 m)=2 m^{\prime}$ if and only if $\varphi\left(b_{m}(X)\right)=b_{m^{\prime}}\left(X^{\prime}\right)$,

- $\psi(2 m+1)=2 m^{\prime}+1$ if and only if $\varphi$ maps $h_{m}(X)$ onto $h_{m^{\prime}}\left(X^{\prime}\right)$.

The function $\psi$ is an isomorphism between $\Phi(X)$ and $\Phi\left(X^{\prime}\right)$.

Conversely, assume $\psi: \mathbb{N} \rightarrow \mathbb{N}$ is an isomorphism between $\Phi(X)$ and $\Phi\left(X^{\prime}\right)$. Since $\psi$ preserves $P, n$ and $\psi(n)$ have the same parity. In order to define a homeomorphism $\varphi: X \rightarrow X^{\prime}$ we first take care of the branch points of $X$ and $X^{\prime}$ setting $\varphi\left(b_{n}(X)\right)=b_{n^{\prime}}\left(X^{\prime}\right)$ for each $n$, where $n^{\prime}=\frac{\psi(2 n)}{2}$.

Claim. The function $\varphi$ so far defined is uniformly continuous on $B(X)$.

Proof of the Claim. If this were not the case there exists $\varepsilon>0$ such that for all $\delta>0$ there exists $x, y \in B(X)$ with $d(x, y)<\delta$ and $d(\varphi(x), \varphi(y))>\varepsilon$. Hence there are two sequences $\left(x_{k}\right)$ and $\left(y_{k}\right)$ in $B(X)$ which converge to the same point in $X$ and such that $\left(\varphi\left(x_{k}\right)\right)$ and $\left(\varphi\left(y_{k}\right)\right)$ converge to distinct points $x^{\prime}, y^{\prime} \in X^{\prime}$. Then $A_{x^{\prime} y^{\prime}}^{X^{\prime}}$ either contains some $h_{n}(X)$ or infinitely many branch points. If $m_{k}^{\prime}$ and $n_{k}^{\prime}$ are the representatives of $\left\{\varphi\left(x_{k}\right)\right\}$ and $\left\{\varphi\left(y_{k}\right)\right\}$ in $\Phi\left(X^{\prime}\right)$, then either for some $j \notin P^{\Phi\left(X^{\prime}\right)}$ we eventually have $\left(m_{k}^{\prime}, j, n_{k}^{\prime}\right) \in R^{\Phi\left(X^{\prime}\right)}$, or for some distinct $j$ and $j^{\prime}$ we eventually have both $\left(m_{k}^{\prime}, j, n_{k}^{\prime}\right) \in R^{\Phi\left(X^{\prime}\right)}$ and $\left(m_{k}^{\prime}, j^{\prime}, n_{k}^{\prime}\right) \in R^{\Phi\left(X^{\prime}\right)}$. On the other hand, if $m_{k}=\psi^{-1}\left(m_{k}^{\prime}\right)$ and $n_{k}=\psi^{-1}\left(n_{k}^{\prime}\right)$, neither does there exist $j \notin P^{\Phi(X)}$ such that eventually $\left(m_{k}, j, n_{k}\right) \in R^{\Phi(X)}$ nor do there exist distinct $j$ and $j^{\prime}$ such that eventually $\left(m_{k}, j, n_{k}\right) \in R^{\Phi(X)}$ and $\left(m_{k}, j^{\prime}, n_{k}\right) \in R^{\Phi(X)}$. This contradicts $\psi$ being an isomorphism.

Therefore $\varphi$ can be extended to a continuous function on $\overline{B(X)}$ and applying the claim to $\psi^{-1}$ as well, we have that $\varphi$ is a homeomorphism between $\overline{B(X)}$ and $\overline{B\left(X^{\prime}\right)}$.

For each $n$, we will define $\varphi$ on $h_{n}(X)$ to be a homeomorphism onto $h_{n^{\prime}}\left(X^{\prime}\right)$ where $n^{\prime}=\frac{\psi(2 n+1)-1}{2}$. Note that at least one end point of $h_{n}(X)$ belongs to $\overline{B(X)}$ while the other end point of $h_{n}(X)$ can be either in $\overline{B(X)}$ or in $E(X)$. Since $\psi$ preserves $R$, the same situation holds for $h_{n^{\prime}}\left(X^{\prime}\right)$. So define $\varphi$ on $h_{n}(X)$ as a homeomorphism onto $h_{n^{\prime}}\left(X^{\prime}\right)$ extending the definition already given on $\overline{B(X)}$.

The fact that $\varphi: X \rightarrow X^{\prime}$ is a homeomorphism follows by again using that $\psi$ is an isomorphism.

Lemma 6.6. The isomorphism relation for countable linear orderings Borel reduces to the homeomorphism relation on $\mathcal{Q}\left(C_{\infty}\right)$.

Proof. Let $\mathcal{L}$ be the language with a single binary relation symbol and let $\mathrm{LO} \subseteq X_{\mathcal{L}}$ be the Polish subspace of countable linear orderings. We will define a continuous function $F: \mathrm{LO} \rightarrow \mathrm{C}(I \times[-1,1])$, reducing isomorphism of countable total orderings to homeomorphism of subcontinua of $I \times[-1,1]$, so that $F(\alpha)$ is quasihomeomorphic to $C_{\infty}$ for every $\alpha \in \mathrm{LO}$.

Let $K=(I \times\{0\}) \cup\left(\left\{\frac{1}{4}\right\} \times\left[-\frac{1}{4}, 1\right]\right)$ and fix an enumeration $\left\{q_{n} \mid n \in \mathbb{N}\right\}$ of the rationals of the open interval $\left(\frac{1}{2}, \frac{3}{4}\right)$. If $\alpha \in \mathrm{LO}$, the continuum $F(\alpha)$ will be obtained by adding to $K$ vertical segments of the form $\left\{q_{n}\right\} \times\left[0, \frac{1}{h}\right]$, where $(n, h)$ ranges in a suitable subset of $\mathbb{N}^{2}$, dependent on $\alpha$ (see Figure 3 where the thicker lines represent $K)$. Note that Corollary 3.8 guarantees that $F(\alpha)$ is quasi- 


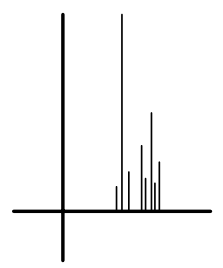

Figure 3. The construction of the proof of Lemma 6.6

homeomorphic to $C_{\infty}$.

Fix $\alpha \in$ LO. We define by recursion $\alpha^{*}: \mathbb{N} \rightarrow \mathbb{N}$. Let $n \in \mathbb{N}$ and suppose $\alpha^{*}(i)$ has been defined for every $i<n$. Then $\alpha^{*}(n)$ is the least $m \in \mathbb{N}$ such that the $n+1$ tuple of rationals $\left(q_{\alpha^{*}(0)}, q_{\alpha^{*}(1)}, \ldots, q_{\alpha^{*}(n-1)}, q_{m}\right)$ is ordered within $I$ as the $n+1$ tuple $(0,1, \ldots, n-1, n)$ is ordered according to $\alpha$. Then $Q_{\alpha}=\left\{q_{\alpha^{*}(n)} \mid n \in \mathbb{N}\right\}$ is order isomorphic to $\alpha$. Moreover, if $J$ is a nonempty interval of $\left(\frac{1}{2}, \frac{3}{4}\right)$ disjoint from $Q_{\alpha}$, then either there are no elements of $Q_{\alpha}$ to the left of $J$ or there is a biggest one; similarly, either there is no member of $Q_{\alpha}$ to the right of $J$ or there is a least one.

We let

$$
F(\alpha)=K \cup \bigcup_{n \in \mathbb{N}}\left(\left\{q_{\alpha^{*}(n)}\right\} \times\left[0, \frac{1}{n+1}\right]\right) .
$$

It is immediate to check that $F$ is continuous.

Assume that $\alpha, \beta \in \mathrm{LO}$ are isomorphic and let $\varphi: \mathbb{N} \rightarrow \mathbb{N}$ be an order isomorphism from $\alpha$ to $\beta$. This induces an order isomorphism $\psi: Q_{\alpha} \rightarrow Q_{\beta}$ defined by $\psi\left(q_{\alpha^{*}(n)}\right)=q_{\beta^{*}(\varphi(n))}$. By the above observation on open intervals disjoint from $Q_{\alpha}, \psi$ can be extended to an order isomorphism (so a homeomorphism) $\psi^{\prime}: I \rightarrow I$ which is the identity on $\left[0, \frac{1}{4}\right]$. Since $\psi^{\prime}$ matches branch points of $F(\alpha)$ with branch points of $F(\beta)$ and obviously $\psi^{\prime}\left(\frac{1}{4}\right)=\frac{1}{4}$, we can extend $\psi^{\prime}$ to a homeomorphism between $F(\alpha)$ and $F(\beta)$.

Conversely, any homeomorphism $\chi$ between $F(\alpha)$ and $F(\beta)$ matches branch points and preserves their ordering on the arc containing all of them. As $\left(\frac{1}{4}, 0\right)$ is the unique point of order 4 in both $F(\alpha)$ and $F(\beta)$, and there are no branch points to its left, $\chi\left(\frac{1}{4}, 0\right)=\left(\frac{1}{4}, 0\right)$ and $\chi$ respects the orientation of $I$. Therefore the restriction of $\chi$ to $Q_{\alpha}$ is an order isomorphism between $Q_{\alpha}$ and $Q_{\beta}$. Hence $\alpha$ and $\beta$ are isomorphic total orderings.

Theorem 6.7. The homeomorphism relation is $S_{\infty}$-universal both on dendrites and on $\mathcal{Q}\left(C_{\infty}\right)$.

Proof. By Lemmas [6.5] and [6.6] and the result of Friedman and Stanley ([FS89]) that isomorphism for countable total orderings is $S_{\infty}$-universal.

Theorem 6.7 solves the classification problem for homeomorphism on some particular classes of compact metric spaces. As already mentioned, homeomorphism on compact subsets of the Cantor space shares the same complexity, while homeomorphism on Peano continua is strictly more complicated.

Corollary 6.8. $\mathcal{Q}\left(C_{\infty}\right)$ contains a perfect set of pairwise non-homeomorphic continua. Moreover, for every $\alpha<\omega_{1}$ there exists a continuum $C \in \mathcal{Q}\left(C_{\infty}\right)$ such 
that $\mathcal{H}(C)$ is not $\boldsymbol{\Pi}_{\alpha}^{0}$, i.e. $\mathcal{Q}\left(C_{\infty}\right)$ is partitioned into homeomorphism classes of unbounded Borel complexity.

Proof. By Theorem 6.7 and the argument of the proof of the second half of Theorem 1.3 .

\section{Constructions for hardness}

We recall a few sets which will be useful (for details see Kec95. §23.A]). They are subsets of the Cantor space $2^{\mathbb{N}}$, sometimes viewed as $2^{\mathbb{N} \times \mathbb{N}}$ or $\left(2^{\mathbb{N} \times \mathbb{N}}\right)^{2}$. The quantifier $\forall^{\infty} n$ means "for all but finitely many $n$ 's", i.e. $\exists M \forall n>M$.

$$
\begin{aligned}
& Q_{2}=\left\{\alpha \in 2^{\mathbb{N}} \mid \forall^{\infty} n \alpha(n)=0\right\} \quad \text { is } \boldsymbol{\Sigma}_{2}^{0} \text {-complete; } \\
& P_{3}=\left\{\alpha \in 2^{\mathbb{N} \times \mathbb{N}} \mid \forall m \forall^{\infty} n \alpha(m, n)=0\right\} \quad \text { is } \boldsymbol{\Pi}_{3}^{0} \text {-complete; } \\
& S_{3}^{*}=\left\{\alpha \in 2^{\mathbb{N} \times \mathbb{N}} \mid \forall^{\infty} m \exists n \alpha(m, n)=0\right\} \quad \text { is } \boldsymbol{\Sigma}_{3}^{0} \text {-complete. }
\end{aligned}
$$

The last two facts imply that $P_{3} \times S_{3}^{*} \subset\left(2^{\mathbb{N} \times \mathbb{N}}\right)^{2}$ is $\mathrm{D}_{2}\left(\boldsymbol{\Sigma}_{3}^{0}\right)$-complete.

The following notation will be useful in our constructions: when $p, q \in \mathbb{R}^{n}$ for some $n, \overline{p q}$ denotes the straight segment joining $p$ and $q$.

Lemma 7.1. Let $X$ be homeomorphic to $I^{2}$ and let $x$ and $y$ be distinct points on the boundary of $X$. There exists a continuous function $F^{\prime}: 2^{\mathbb{N}} \rightarrow \mathrm{C}(X)$ such that

(1) $F^{\prime}(\alpha)$ intersects the boundary of $X$ in the set $\{x, y\}$ for every $\alpha \in 2^{\mathbb{N}}$;

(2) if $\alpha \in Q_{2}$, then $F^{\prime}(\alpha)$ is an arc with end points $x$ and $y$;

(3) if $\alpha \notin Q_{2}$, then $F^{\prime}(\alpha)$ is not a Peano continuum.

Proof. We can assume $X=[-1,1]^{2}, x=(1,0)$ and $y=(0,1)$. For every $n$ let $p_{n}=\left(\frac{1}{n+1}, 0\right), q_{n}=\left(\frac{1}{n+2}, \frac{1}{2}\right)$ and define $F_{n}^{\prime}(0)=\overline{p_{n} p_{n+1}}$ and $F_{n}^{\prime}(1)=\overline{p_{n} q_{n}} \cup \overline{q_{n} p_{n+1}}$. Also let $p_{\infty}=(0,0)$.

If $\alpha \in 2^{\mathbb{N}}$ let

$$
F^{\prime}(\alpha)=\overline{p_{\infty} y} \cup \bigcup_{n \in \mathbb{N}} F_{n}^{\prime}(\alpha(n))
$$

(see Figure 4). To check that $F^{\prime}: 2^{\mathbb{N}} \rightarrow \mathrm{C}(X)$ is continuous notice that if $\alpha(n)=$ $\beta(n)$ for every $n<m$, then $d_{H}\left(F^{\prime}(\alpha), F^{\prime}(\beta)\right)<\frac{1}{m}$.

The first property of $F^{\prime}$ is immediate from the definition. The second property is checked by noting that if $\alpha \in Q_{2}$, then $\left\{p_{\infty}\right\} \cup \bigcup_{n \in \mathbb{N}} F_{n}^{\prime}(\alpha(n))$ is an arc with end points $x$ and $p_{\infty}$ which intersects $\overline{p_{\infty} y}$ only in $p_{\infty}$. To check the third property note that if $\alpha \notin Q_{2}$, then $F^{\prime}(\alpha)$ is not locally connected at every point of the lower half of $\overline{p_{\infty} y}$.

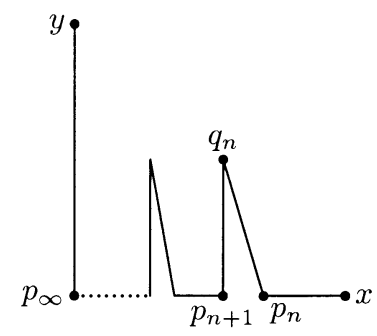

Figure 4. The construction of the proof of Lemma 7.1 


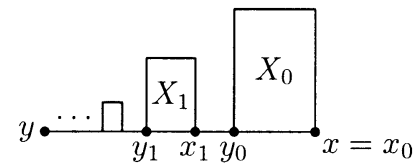

FiguRE 5. The construction of the proof of Lemma 7.2

Lemma 7.1 can be applied repeatedly, yielding the following sharper result.

Lemma 7.2. Let $X$ be homeomorphic to $I^{2}$ and let $x$ and $y$ be distinct points on the boundary of $X$. There exists a continuous function $F: 2^{\mathbb{N} \times \mathbb{N}} \rightarrow \mathrm{C}(X)$ such that

(1) $F(\alpha)$ intersects the boundary of $X$ in the set $\{x, y\}$ for every $\alpha \in 2^{\mathbb{N} \times \mathbb{N}}$;

(2) if $\alpha \in P_{3}$, then $F(\alpha)$ is an arc with end points $x$ and $y$;

(3) if $\alpha \notin P_{3}$, then $F(\alpha)$ is not a Peano continuum.

Proof. We can assume $X=[0,1] \times[-1,1], x=(1,0)$ and $y=(0,0)$. For every $m$ let $X_{m}=\left[\frac{1}{2 m+2}, \frac{1}{2 m+1}\right] \times\left[0, \frac{1}{m+1}\right], x_{m}=\left(\frac{1}{2 m+1}, 0\right)$ and $y_{m}=\left(\frac{1}{2 m+2}, 0\right)$ (see Figure 5). Let $F_{m}^{\prime}: 2^{\mathbb{N}} \rightarrow \mathrm{C}\left(X_{m}\right)$ be the continuous function provided by Lemma 7.1 with respect to $x_{m}$ and $y_{m}$.

If $\alpha \in 2^{\mathbb{N} \times \mathbb{N}}$ let $\alpha_{m} \in 2^{\mathbb{N}}$ be defined by $\alpha_{m}(n)=\alpha(m, n)$ and define

$$
F(\alpha)=\{y\} \cup \bigcup_{m \in \mathbb{N}}\left(F_{m}^{\prime}\left(\alpha_{m}\right) \cup \overline{y_{m} x_{m+1}}\right) .
$$

To check that $F: 2^{\mathbb{N} \times \mathbb{N}} \rightarrow \mathrm{C}(X)$ is continuous notice that if $d_{H}\left(F_{m}^{\prime}\left(\alpha_{m}\right), F_{m}^{\prime}\left(\beta_{m}\right)\right)<$ $\frac{1}{M}$ for every $m<M$, then $d_{H}(F(\alpha), F(\beta))<\frac{1}{M}$.

The first property of $F$ is immediate from the definition. The second property is checked by noting that if $\alpha \in P_{3}$, then each $F_{m}^{\prime}\left(\alpha_{m}\right)$ is an arc with end points $x_{m}$ and $y_{m}$ contained in $X_{m}$. To check the third property notice that if $\alpha \notin P_{3}$, then $F_{m}^{\prime}\left(\alpha_{m}\right)$ is not locally connected for some $m$; by the choice of the $X_{m}$ 's, $F(\alpha)$ is also not locally connected.

Lemma 7.2 immediately yields a hardness result for a wide range of classes of Peano continua.

Theorem 7.3. Any class of Peano continua which is closed under homeomorphism and contains a continuum which has an open free arc is $\boldsymbol{\Pi}_{3}^{0}$-hard.

Proof. Let $P \in \mathrm{C}\left(I^{\mathbb{N}}\right)$ be a Peano continuum which contains an open free $\operatorname{arc} A$. Fix distinct points $x$ and $y$ belonging to $A$ and let $B \subset A$ be the arc with end points $x$ and $y$. Let $P^{\prime}=P \backslash B$.

Since $A$ is open in $P$, there exists $X$ homeomorphic to $I^{2}$ such that $X \cap P=$ $B$, and $x$ and $y$ are the only points of $B$ which lie on the boundary of $X$. Let $F: 2^{\mathbb{N} \times \mathbb{N}} \rightarrow \mathrm{C}(X)$ be the function of Lemma 7.2 with respect to $x$ and $y$. Define $\bar{F}: 2^{\mathbb{N} \times \mathbb{N}} \rightarrow \mathrm{C}\left(I^{\mathbb{N}}\right)$ by $\bar{F}(\alpha)=P^{\prime} \cup F(\alpha)$.

Since $d_{H}(\bar{F}(\alpha), \bar{F}(\beta)) \leq d_{H}(F(\alpha), F(\beta))$ the function $\bar{F}$ is continuous. Moreover it is immediate that

$$
\begin{aligned}
\alpha \in P_{3} & \Longleftrightarrow \bar{F}(\alpha) \text { is homeomorphic to } P \\
& \Longleftrightarrow \bar{F}(\alpha) \in \mathcal{P} .
\end{aligned}
$$



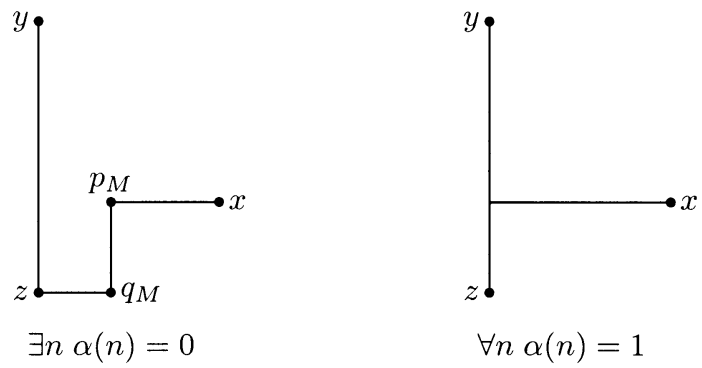

Figure 6 . The construction of the proof of Lemma 7.4

If $\mathcal{C}$ is any class of Peano continua satisfying the hypothesis of the theorem pick $P \in \mathcal{C}$ with an open free arc, and apply the construction above. We have

$$
\alpha \in P_{3} \Longleftrightarrow \bar{F}(\alpha) \in \mathcal{C},
$$

and $\mathcal{C}$ is $\Pi_{3}^{0}$-hard.

Another useful construction is contained in the next lemma.

Lemma 7.4. Let $X$ be homeomorphic to $I^{2}$ and let $x$ and $y$ be distinct points on the boundary of $X$. There exists a continuous function $G: 2^{\mathbb{N}} \rightarrow \mathrm{C}(X)$ such that

(1) $G(\alpha)$ intersects the boundary of $X$ in the set $\{x, y\}$ for every $\alpha \in 2^{\mathbb{N}}$;

(2) if $\forall n \alpha(n)=1$, then $G(\alpha)$ is a simple triod such that $x$ and $y$ are two of its end points;

(3) if $\exists n \alpha(n)=0$, then $G(\alpha)$ is an arc with end points $x$ and $y$.

Proof. We can assume $X=[-1,1]^{2}, x=(1,0)$ and $y=(0,1)$. Let $z=\left(0,-\frac{1}{2}\right)$ and, for every $n, p_{n}=\left(\frac{1}{n+1}, 0\right)$ and $q_{n}=\left(\frac{1}{n+1},-\frac{1}{2}\right)$.

If $\alpha \in 2^{\mathbb{N}}$ let $A_{\alpha}=\{n \mid \forall m<n \alpha(m)=1\}$. If $\exists n \alpha(n)=0$ let $M=\max A_{\alpha}+1$ and $E_{\alpha}=\overline{p_{M} q_{M}}$. If $\forall n \alpha(n)=1$ let $E_{\alpha}=\emptyset$. Define $G$ by

$$
G(\alpha)=\overline{y z} \cup E_{\alpha} \cup \bigcup_{n \in A_{\alpha}} \overline{p_{n} p_{n+1}} \cup \bigcup_{n \notin A_{\alpha}} \overline{q_{n} q_{n+1}}
$$

(see Figure 6). To check that $G: 2^{\mathbb{N}} \rightarrow \mathrm{C}(X)$ is continuous notice that if $\alpha(n)=$ $\beta(n)$ for every $n<m$, then $d_{H}(G(\alpha), G(\beta))<\frac{1}{m}$.

The three properties $G$ must satisfy are easily checked.

Combining Lemmas 7.1 and 7.4 we obtain the following result.

Theorem 7.5. Any class of graphs which contains all combs is $\mathrm{D}_{2}\left(\boldsymbol{\Sigma}_{3}^{0}\right)$-hard.

Proof. Let $X=[0,1] \times[-1,1]$ and, for every $m, X_{m}=\left[\frac{1}{2 m+2}, \frac{1}{2 m+1}\right] \times\left[0, \frac{1}{m+1}\right]$, $x_{m}=\left(\frac{1}{2 m+1}, 0\right)$ and $y_{m}=\left(\frac{1}{2 m+2}, 0\right)$ as in the proof of Lemma 7.2 (see again Figure [5).

When $m$ is even let $F_{m}^{\prime}: 2^{\mathbb{N}} \rightarrow \mathrm{C}\left(X_{m}\right)$ be the continuous function provided by Lemma 7.1 with respect to $x_{m}$ and $y_{m}$. When $m$ is odd let $G_{m}: 2^{\mathbb{N}} \rightarrow \mathrm{C}\left(X_{m}\right)$ be the continuous function provided by Lemma 7.4 with respect to $x_{m}$ and $y_{m}$. 
If $(\alpha, \beta) \in\left(2^{\mathbb{N} \times \mathbb{N}}\right)^{2}$ let $\alpha_{m}, \beta_{m} \in 2^{\mathbb{N}}$ be defined as in the proof of Lemma 7.2 Define $\bar{F}:\left(2^{\mathbb{N} \times \mathbb{N}}\right)^{2} \rightarrow \mathrm{C}(X)$ by

$$
\bar{F}(\alpha, \beta)=\{(0,0)\} \cup \bigcup_{m \in \mathbb{N}}\left(F_{2 m}^{\prime}\left(\alpha_{m}\right) \cup \overline{y_{2 m} x_{2 m+1}} \cup G_{2 m+1}\left(\beta_{m}\right) \cup \overline{y_{2 m+1} x_{2 m+2}}\right) .
$$

The continuity of $\bar{F}$ is proved as in the proof of Lemma 7.2

If $\alpha \notin P_{3}$, then $\bar{F}(\alpha, \beta)$ is not a Peano continuum (and thus not a graph) because some $F_{2 m}^{\prime}\left(\alpha_{m}\right)$ is not a Peano continuum. If $\beta \notin S_{3}^{*}$, then infinitely many $G_{2 m+1}\left(\beta_{m}\right)$ 's are simple triods, so that $\bar{F}(\alpha, \beta)$ has infinitely many branching points and hence is not a graph. If $(\alpha, \beta) \in P_{3} \times S_{3}^{*}$, then each $F_{2 m}^{\prime}\left(\alpha_{m}\right)$ is an arc and all but finitely many $G_{2 m+1}\left(\beta_{m}\right)$ 's are arcs, so that $\bar{F}(\alpha, \beta)$ is a comb (the number of branching points is the number of $m$ 's such that $\forall n \beta(m, n)=1)$.

Therefore

$$
\begin{aligned}
(\alpha, \beta) \in P_{3} \times S_{3}^{*} & \Longleftrightarrow \bar{F}(\alpha, \beta) \text { is a comb } \\
& \Longleftrightarrow \bar{F}(\alpha, \beta) \text { is a graph }
\end{aligned}
$$

and this completes the proof of the theorem.

\section{Classification of Classes of continua}

For the sake of completeness, we begin with a list of some $\boldsymbol{\Pi}_{2}^{0}$-complete classes of continua. For some of them the classification is already known, for others it follows from simple computations.

Theorem 8.1. The following classes of continua are $\boldsymbol{\Pi}_{2}^{0}$-complete:

(1) pseudoarcs;

(2) indecomposable continua;

(3) hereditarily indecomposable continua;

(4) irreducible continua between $n$ points $(n \geq 2)$;

(5) hereditarily irreducible continua;

(6) unicoherent continua;

(7) hereditarily unicoherent continua.

Proof. Once we show that each class is $\boldsymbol{\Pi}_{2}^{0}$, its completeness follows from the Baire Category Theorem since it is dense with dense complement. The proof that a class is $\Pi_{2}^{0}$ is a simple check on its definition or one of its equivalent characterizations.

(1) See [Nad92, exercise 12.70].

(2) See [Nad92, exercise 1.17].

(3) See [Nad92, exercise 1.23].

(4) A theorem of Sorgenfrey ([Sor46]) states that a continuum $X$ is irreducible about $n$ points $(n \geq 2)$ if and only if, whenever $X=\bigoplus_{i=0}^{n} X_{i}$, there exists $j \leq n$ such that $\bigcup_{i \neq j} X_{i}$ is not connected.

(5) Follows immediately from (4).

(6) A continuum $X \subseteq I^{\mathbb{N}}$ is unicoherent if and only if

$$
\forall A, B \in \mathrm{C}\left(I^{\mathbb{N}}\right)\left(A, B \subseteq X \& A \cup B=X \Longrightarrow A \cap B \in \mathrm{C}\left(I^{\mathbb{N}}\right)\right) .
$$

Since intersection is a Baire class 1 function on $\mathrm{K}\left(I^{\mathbb{N}}\right)$ (see [Kur68]), this formula defines a $\Pi_{2}^{0}$ set.

(7) Follows immediately from (6). 
We now list classifications of projective classes of continua that are already known or follow immediately from already known constructions.

Theorem 8.2. (1) The class of arcwise connected continua is $\mathbf{\Pi}_{2}^{1}$-complete;

(2) the class of hereditarily decomposable continua is $\boldsymbol{\Pi}_{1}^{1}$-complete;

(3) the class of continua that do not contain any hereditarily indecomposable subcontinuum is $\boldsymbol{\Pi}_{1}^{1}$-complete;

(4) the class of $\lambda$-dendroids is $\boldsymbol{\Pi}_{1}^{1}$-complete.

Proof. (1) This is due to Ajtai and Becker (see Kec95. Theorem 37.11]).

(2) This is proved in Dar00.

(3) To see that the class under consideration is $\Pi_{1}^{1}$ it suffices to check the definition, applying Theorem 8.13, $\mathbf{\Pi}_{1}^{1}$-hardness can be proved with the construction of [Dar00].

(4) The class of $\lambda$-dendroids is $\Pi_{1}^{1}$ by Theorem 8.17 and (2). $\Pi_{1}^{1}$-hardness is proved again by the construction of [Dar00].

From Theorem 7.3 and Lemma 6.1 we have the following (for the class of Peano continua this was first obtained by Kuratowski ([Kur31]) and Mazurkiewicz (Maz31])).

Theorem 8.3. The classes $\mathcal{P}$ of Peano continua and $\mathcal{D}$ of dendrites are $\boldsymbol{\Pi}_{3}^{0}$ complete.

Theorem 8.4. Each of the classes of graphs and trees is $\mathrm{D}_{2}\left(\boldsymbol{\Sigma}_{3}^{0}\right)$-complete.

Proof. Both classes are $\mathrm{D}_{2}\left(\boldsymbol{\Sigma}_{3}^{0}\right)$-hard by Theorem 7.5

It is obvious that there are countably many homeomorphism classes of graphs. Let $\left\{G_{n} \mid n \in \mathbb{N}\right\}$ be a set of graphs intersecting each homeomorphism class of graphs. By Theorem 1.7 the class of graphs is

$$
\mathcal{P} \cap \bigcup_{n \in \mathbb{N}} \mathcal{L}_{G_{n}} .
$$

Theorem 8.3 and Corollary 5.4 imply that the class of graphs is $\mathrm{D}_{2}\left(\boldsymbol{\Sigma}_{3}^{0}\right)$.

The proof that the class of trees is $\mathrm{D}_{2}\left(\boldsymbol{\Sigma}_{3}^{0}\right)$ is analogous, starting with a countable set of trees intersecting each homeomorphism class of trees.

By combining the results of Sections 3 and 7 we can now show that the classification of the complexity of classes of quasi-homeomorphism of dendrites obtained in Lemma 6.2 is sharp.

Theorem 8.5. If $D$ is a nondegenerate dendrite, then the class $\mathcal{Q}(D)$ is $\boldsymbol{\Pi}_{3}^{0}$ complete.

Proof. $\mathcal{Q}(D)$ is $\Pi_{3}^{0}$ by Lemma 6.2 .

Since not all nondegenerate dendrites contain an open free arc, Theorem 7.3 cannot be applied directly. However we now show that every quasi-homeomorphism class of dendrites has an element with an open free arc and hence is $\boldsymbol{\Pi}_{3}^{0}$-hard.

Given $D$ fix $p \in E(D)$ and set $D^{\prime}=D \cup A$ where $A$ is an arc with $p$ as one of its end points and $A \cap D=\{p\}$. Clearly $D^{\prime}$ contains an open free $\operatorname{arc}$ and $D \preceq D^{\prime}$. Since any subtree of $D^{\prime}$ is homeomorphic to a subtree of $D$, Theorem 3.2(4) shows that $D^{\prime} \preceq D$. Therefore $D^{\prime} \in \mathcal{Q}(D)$. 
Corollary 8.6. If $D$ is a dendrite with finitely many branch points, then the class $\mathcal{H}(D)$ is $\boldsymbol{\Pi}_{3}^{0}$-complete.

Proof. By Theorems 3.5 and 8.5

Corollary 6.8 shows that Corollary 8.6 cannot be extended to arbitrary dendrites. The technique of the proof of Lemma 6.2 applies also to graphs.

Theorem 8.7. If $G$ is a graph, then the class $\mathcal{Q}(G)=\mathcal{H}(G)$ is $\Pi_{3}^{0}$-complete.

Proof. The two classes coincide by Theorem 1.6 and are $\boldsymbol{\Pi}_{3}^{0}$-hard by Theorem 7.3

Since there are only countably many classes of homeomorphism of graphs, there exists a countable set of graphs $\left\{G_{n} \mid n \in \mathbb{N}\right\}$ such that $\forall n G_{n} \prec G$ and $\forall G^{\prime} \prec$ $G \exists n \mathcal{H}\left(G^{\prime}\right)=\mathcal{H}\left(G_{n}\right)$. We have again

$$
\mathcal{Q}(G)=\left(\mathcal{P} \cap \mathcal{L}_{G}\right) \backslash \bigcup_{n \in \mathbb{N}} \mathcal{L}_{G_{n}}
$$

and Theorem 8.3 and Corollary 5.4 imply that $\mathcal{Q}(G)$ is $\Pi_{3}^{0}$.

In particular, each of the homeomorphism classes of the arc (this already follows from [Kur31 and Maz31]), the simple triod and the circle is $\boldsymbol{\Pi}_{3}^{0}$-complete.

Theorem 8.8. The classes of uniquely arcwise connected continua and of dendroids are $\boldsymbol{\Pi}_{1}^{1}$-complete.

Proof. Let $\mathcal{R}$ be the set of $(C, A, x, y) \in \mathrm{C}\left(I^{\mathbb{N}}\right)^{2} \times\left(I^{\mathbb{N}}\right)^{2}$ such that $A \subseteq C$ is an arc with end points $x$ and $y$. Then a continuum $C$ is uniquely arcwise connected if and only if

$$
\forall x, y \in I^{\mathbb{N}}\left(x, y \in C \& x \neq y \Longrightarrow \exists ! A \in \mathrm{C}\left(I^{\mathbb{N}}\right)(C, A, x, y) \in \mathcal{R}\right) .
$$

A straightforward computation using Corollary 8.6 shows that $\mathcal{R}$ is $\Pi_{3}^{0}$ and hence (by a well-known result of descriptive set theory; see [Kec95, Theorem 18.11]) the class of uniquely arcwise connected continua is $\Pi_{1}^{1}$. A continuum is a dendroid if and only if it is hereditarily unicoherent and uniquely arcwise connected. By Theorem 8.1(7) and the first part of the theorem the class of dendroids is $\boldsymbol{\Pi}_{1}^{1}$.

The construction of [Dar00] shows that both classes are $\boldsymbol{\Pi}_{1}^{1}$-hard.

\section{REFERENCES}

[Ale28] Paul Alexandroff, Über den allgemeinen Dimensionsbegriff und seine Beziehungen zur elementaren geometrischen Anschauung, Math. Ann. 98 (1928), 617-635.

[Bin51] R. H. Bing, Concerning hereditarily indecomposable continua, Pacific J. Math. 1 (1951), 43-51. MR0043451 (13,265b)

[BK96] Howard Becker and Alexander S. Kechris, The descriptive set theory of Polish group actions, Cambridge University Press, Cambridge, 1996. MF 1425877 (98d:54068)

[CG01] Riccardo Camerlo and Su Gao, The completeness of the isomorphism relation for countable Boolean algebras, Trans. Amer. Math. Soc. 353 (2001), no. 2, 491-518. MR 1804507 (2001k:03097)

[Dar00] Udayan B. Darji, Complexity of hereditarily decomposable continua, Topology Appl. 103 (2000), no. 3, 243-248. MR1758437 (2001a:54044)

[DM04] Udayan B. Darji and Alberto Marcone, Complexity of curves, Fund. Math. 182 (2004), no. 1, 79-93. MR2100716

[FS89] Harvey Friedman and Lee Stanley, A Borel reducibility theory for classes of countable structures, J. Symbolic Logic 54 (1989), no. 3, 894-914. MR1011177 (91f:03062)

[Hjo00] Greg Hjorth, Classification and orbit equivalence relations, American Mathematical Society, Providence, RI, 2000. MR.1725642 [2000k:03097) 
[Kec95] Alexander S. Kechris, Classical descriptive set theory, Graduate Texts in Mathematics, no. 156, Springer-Verlag, New York, 1995. MR.1321597 (96e:03057)

[Kec02] _ Actions of Polish groups and classification problems, Analysis and logic (Mons, 1997), London Math. Soc. Lecture Note Ser., vol. 262, Cambridge Univ. Press, Cambridge, 2002, pp. 115-187. MR1967835 (2004b:03070)

[Kur31] Kazimierz Kuratowski, Évaluation de la classe borélienne ou projective d'un ensemble de points à l'aide des symboles logiques, Fund. Math. 17 (1931), 249-272.

[Kur68] — Topology. Vol. II, Academic Press, New York, 1968. MR0259835 (41:4467)

[KY00] Hisao Kato and Xiangdong Ye, On Burgess's theorem and related problems, Proc. Amer. Math. Soc. 128 (2000), no. 8, 2501-2506. MR.1653402|(2000k:54008)

[Lav71] Richard Laver, On Fraïssé's order type conjecture, Ann. of Math. (2) 93 (1971), 89-111. MR.0279005 (43:4731)

[Maz31] Stefan Mazurkiewicz, Sur l'ensemble des continus péaniens, Fund. Math. 17 (1931), 273-274.

[Mil85] Eric C. Milner, Basic wqo- and bqo-theory, Graphs and order (Ivan Rival, ed.), D. Reidel, Dordrecht, 1985, pp. 487-502. MR0818505 (87h:04004)

[MR04] Alberto Marcone and Christian Rosendal, The complexity of continuous embeddability between dendrites, J. Symbolic Logic 69 (2004), no. 3, 663-673. MR.2078915 (2005e:03106)

[Nad92] Sam B. Nadler, Jr., Continuum theory, Marcel Dekker Inc., New York, 1992. MR1192552 (93m:54002)

[RN65] C. Ryll-Nardzewski, On a Freedman's problem, Fund. Math. 57 (1965), 273-274. MR:1192552 (93m:54002)

[Sim85] Stephen G. Simpson, Bqo-theory and Fraïssé's conjecture, Recursive aspects of descriptive set theory, Oxford University Press, New York, 1985, by Richard Mansfield and Galen Weitkamp, pp. 124-138.

[Sor46] R. H. Sorgenfrey, Concerning continua irreducible about $n$ points, Amer. J. Math. 68 (1946), 667-671. MR0017523 (8:165c)

[vM89] Jan van Mill, Infinite-dimensional topology, North-Holland Publishing Co., Amsterdam, 1989. MR0977744 (90a:57025)

Dipartimento di Matematica, Politecnico di Torino, Corso Duca degli Abruzzi 24, 10129 TORINO, ITALY

E-mail address: camerlo@calvino.polito.it

Department of Mathematics, 224 Natural Sciences Building, University of Louisville, Louisville, KentuCKy 40292

E-mail address: ubdarj01@athena.louisville.edu

Dipartimento di Matematica e Informatica, Università di Udine, Via delle Scienze 208, 33100 Udine, ITALY

E-mail address: marcone@dimi.uniud.it 